\title{
Analysis of high-order sub-harmonically injection-locked oscillators
}

\author{
Silvia Hernández ${ }^{1}$, Mabel Pontón ${ }^{1}$, Sergio Sancho ${ }^{1}$, Almudena Suárez ${ }^{1}$ \\ ${ }^{1}$ Departamento de Ingeniería de Comunicaciones, Universidad de Cantabria, Spain
}

High-order subharmonically injection-locked oscillators have recently been proposed for low phase-noise frequency generation, with carrier-selection capabilities. Though excellent experimental behaviour has been demonstrated, the analysis/simulation of these circuits is demanding, due to the high ratio between the oscillation frequency and the frequency of the input source. This work provides an analysis methodology that covers the main aspects of the circuit behaviour, including the detection of the locking bands and the prediction of the phasenoise spectral density. Initially, the oscillator in the presence of a multi-harmonic input source is described with a reduced-order envelope-domain formulation, at the oscillation frequency, based on an oscillator-admittance function extracted from harmonic-balance simulations. This allows deriving an expression for the oscillation phase shift with respect to the input source, and the average of this phase shift is shown to evolve in a continuous manner in the distinct synchronization bands obtained when varying a tuning voltage. This property can be used to detect the locking bands in circuit-level envelope-domain simulations, which, as shown here, can be done through different Fourier decompositions and sampling rates. The phase noise of the high-order subharmonic injection locked oscillator under an arbitrary periodic input waveform is investigated in detail. The frequency response to the noise sources is described with a semi-analytical formulation, relying on the oscillator-admittance function in injectionlocked conditions. The input noise is derived from the timing jitter of the injection source and the phase-noise response is shown to exhibit a low-pass characteristic, which initially follows the up converted input noise and then the oscillator own noise sources. A method is proposed to identify the key parameters of the derived phase-noise spectrum from envelope-domain simulations. The various analysis methodologies have been applied to a prototype at $2.7 \mathrm{GHz}$ at the subharmonic order $N=30$ which has been manufactured and measured.

Keywords: Authors should not add keywords, as these will be chosen during the submission process (see http://journals.cambridge.org/data/relatedlink/MRF topics.pdf for the full list)

Corresponding author: Mabel Pontón; email:

mabel.ponton@unican.es; 


\section{INTRODUCTION}

The recent works [1]-[7] have demonstrated a novel frequency-synthesis methodology, based on the high-order subharmonic injection-locking of a high frequency oscillator, at a frequency $f_{o}$, by an independent source at a much lower frequency $f_{\text {in. }}$. In [1]-[3], the high ratio $N$ between the two frequencies (in the order of $N=30$ ) is achieved by shaping the input sinusoidal signal into a square signal that periodically switches on and off a high-frequency oscillator. This is used to injection lock a second oscillator that selects the harmonic closest to its own free running frequency. The procedure has two advantages. On the one hand, as demonstrated in [4], the phase-noise spectral density of the higher-frequency oscillator can be lower than the one resulting from lower subharmonic ratios (due to the higher phase-noise of the required input source). On the other hand, the availability of a high number of equally spaced spectral lines enables a programmable carrier generation. Actually, the second injection-locked oscillator in [1]-[3] enables the selection of one or another spectral line (with the frequency spacing $f_{o} / N$ ) depending on its tuning voltage. The whole system has the advantage of a low phase noise in comparison with standard phase-locking methodologies [2], [4].

Successful experimental demonstrations of this promising frequency-synthesis method, as well as several extensions of the new procedure have been presented in [1]-[7]. However, the realistic simulation of the high-order sub-harmonic injection-locked oscillator, required for an accurate prediction of its behaviour, is involved. In general, full time-domain simulations, which often fail in the presence of distributed elements, are only possible with simplified models of the circuit components, as done in [1]-[3], where models of the Van der Pol type are used to describe the transistor devices. In [1]-[3], an approximate analytical formulation in time-domain is proposed, in which the synchronized states are detected through a comparison of the oscillation amplitude and phase values at the beginning and end of each period of the switching signal. On the other hand, though harmonic balance (HB) [8]-[12] can deal at present with a high number of harmonic terms, in injection-locked oscillators it converges by default to the non-oscillatory solution that generally coexists with the oscillatory one. In the non-oscillatory solution the circuit just responds to the input forcing in a non-autonomous manner [13]-[15]. To consider the self-oscillation in commercial HB, one should impose the oscillation condition using an auxiliary generator (AG) [13]-[15]. The AG amplitude and its phase-shift with respect to the input source are optimized to obtain a zero value of the $A G$ total admittance function. In the case of a subharmonic injection locking of a high order $N$, the convergence of this optimization procedure is demanding [15] due to the need of a high number $\mathrm{NH}$ of harmonic terms, involving multiples of the high order $N$. For instance, the consideration of 3 harmonic components of the oscillation frequency $f_{o}$, requires $N H=3 N=90$.

This work is an extension of [15], presenting an envelope-domain analysis [16]-[19] of the sub-harmonic injection-locked oscillator is presented, using a Fourier-series description of the circuit variables, with time-varying harmonic terms $\bar{X}_{k}(t)$, where $k$ is the frequency index. This will require a proper initialization of the oscillation frequency to avoid the system convergence to a non-oscillatory steady-state solution [20]. As will be shown, it is possible to choose different fundamental frequencies, expressed as $f_{o} / P$ where $P$ is an integer. For $P>1$, 
the spectral lines of the subharmonic regime are distributed among the harmonic components $k f_{o} / P$ of the Fourier representation of the circuit variables. Then, even in injection-locked conditions, the harmonic terms $\bar{X}_{k}(t)$ will exhibit time variations due to the spectral lines contained in the bandwidth about each harmonic frequency $k f_{o} / P$, so a criterion is needed for an efficient detection of the synchronization bands. In order to derive this criterion, a semianalytical formulation in the envelope-domain, based on an admittance-type model of the oscillator circuit, will be initially considered. An analytical expression will be derived, demonstrating that the injection-locked states can be efficiently detected through the averaging oscillator phase. In addition, the closed solution curves, characteristic of the injection-locked operation [10]-[13], will be obtained through an averaging of the envelope amplitude.

In the solution of the subharmonic injection-locked oscillator there will be a high number of spectral lines at multiples of the low injection-locking frequency $f_{\text {in }}$. This can be achieved through pulse forming plus oscillator switching, as in [1]-[3], or using of a multi-harmonic input source, as in this work. As proposed in [1]-[3], the selection of a particular spectral line can be carried out connecting the oscillator output to a second oscillator circuit (in the same frequency order) with frequency tuning capabilities. The injection-locking (at the fundamental frequency) of this second oscillator enables the selection of one or another spectral line by varying its tuning voltage. Here, this frequency selection by means of injection locking will be analytically investigated considering an oscillator injected with multiple closely-spaced input tones.

The phase-noise analysis of the high-order sub-harmonic injection-locked oscillator will be addressed here for the first time to our knowledge. This analysis is demanding, since, as stated, the HB analysis will fail to converge in most cases due to the need to fulfil the oscillation condition under a high number of harmonic terms. On the other hand, a Monte-Carlo phasenoise analysis in the envelope domain is virtually impossible, due to the presence of two widely separated time scales, one associated with the noise perturbation and the other one accounting for the bandwidth about each harmonic term. Even for $P=N$, the integration time step required to obtain convergence to the oscillatory solution will generally be much smaller than the total simulation time needed to account for the effect of the noise sources. Here the phase noise will be studied in two different manners. First, the phase-noise spectrum will be analytically derived from a perturbation analysis at the oscillation frequency, relying on an admittance-type model [21] of the oscillator circuit in its injection-locked steady state. This formulation will allow an understanding of the frequency response of the subharmonic injection-locked oscillator with respect to the input-source noise and its own noise sources. Then, a procedure to identify the key parameters defining the spectrum shape from circuit-level envelope-transient simulations will be proposed. The whole methodology will be applied to a subharmonic injection-locked oscillator at the order $N=30$, which has been manufactured and measured.

The paper is organized as follows. In Section II, the response of an oscillator driven by multiple periodic tones with a small frequency spacing will be presented. In Section III, the envelope-domain analysis of a subharmonic injection-locked oscillator under a high order $N$ will be described. Section IV presents the derivation of the phase-noise spectrum in the frequency domain, as well as a procedure to identify the parameters that define this spectrum through envelope-transient simulations. 


\section{Oscillator injected by multiple input tones}

In this section, the case of an oscillator injected with multiple input tones about the oscillation frequency will be studied [15], which is conceptually simpler than the one of a subharmonic injection-locked oscillator under a high order $N$, considered in Section III. This will allow an understanding of the oscillator behaviour when driven by multiple tones and an evaluation of its frequency-selection capabilities. A criterion will be derived to determine the locking ranges about each input tone when a parameter is varied, which will be extended in Section III to the subharmonic injection-locked oscillator.

\section{A) Analytical formulation}

A set of input tones will be introduced into the oscillator circuit, at the frequencies $\omega_{1}, \omega_{2}, \ldots, \omega_{K}$, assumed to be closely spaced about the original free-running oscillation frequency $\omega_{o}$. When varying a suitably chosen tuning parameter $\eta$, the oscillator should be able to lock to each of the different input frequencies $\omega_{k}$. To get analytical insight, the oscillator will be described at its fundamental frequency, in terms of its current-to-voltage ratio $Y(V, \omega, \eta)$ at a particular observation node [22]-[26], where $V$ and $\omega$ are the excitation amplitude and frequency. This admittance function will be the current-to-voltage ration of an auxiliary generator (AG) connected at the observation node in the harmonic balance simulation of the oscillator circuit. In the absence of the input signals, at its free-running solution, the oscillator fulfils the following two-tier equation system [11]:

$$
\begin{aligned}
& Y\left(V_{o}, \omega_{o}, \eta_{o}\right)=0 \\
& \bar{H}\left(\bar{X}^{\prime}, V, \omega, \eta_{o}\right)=0
\end{aligned}
$$

where $\bar{H}$ represents the harmonic balance system that constitutes the inner tier and $V_{o}$ and $\omega_{o}$, are the free-running amplitude and frequency at $\eta_{o}$. Note that the phase origin has been arbitrarily taken at the observation node $V e^{j 0}$ and the vector $\bar{X}^{\prime}$ contains all the state variables except $V e^{j 0}$. The above system can be solved through optimization of $V$ and $\omega$ in order to fulfil the goal $Y=0$.

Now, the effect of the input source will be considered. Injection-locking to the particular input tone $\omega_{q}$, belonging to the set of input tones $\omega_{1}, \omega_{2}, \ldots, \omega_{K}$, will be assumed. The oscillator will be formulated in the envelope domain at the carrier frequency $\omega_{q}$. Thus, the node voltage will be expressed as $\left(V_{o}+\Delta V(t)\right) e^{j \phi(t)} e^{j \omega_{q} t}$, where $\Delta V(t)$ is the time-varying increment of the voltage amplitude with respect to the free-running value $V_{o}$ and $\phi(t)$ is the time-varying phase, in the presence of the input sources. Applying Kirchoff's laws at the observation node one obtains the following system:

$$
Y\left(V_{o}+\Delta V(t), \eta_{o}+\Delta \eta, \omega_{o}+\Delta \omega_{q}+s / j\right)\left(V_{o}+\Delta V(t)\right) e^{j \phi(t)}=\sum_{k} I_{k} \exp j\left(\Delta \omega_{k, q} t\right), \quad \Delta \omega_{k, q}=\omega_{k}-\omega_{q}
$$

where $\Delta \omega_{q}=\omega_{q}-\omega_{o}$ and $s$ is a complex frequency increment and $I_{k}$ is the equivalent complex current associated with each input signal. Note that $\Delta \omega_{k, q}$ is the offset frequency of each input tone $k \neq q$ with respect to $\omega_{q}$. Under a small input power and small frequency spacing between the input tones, the function $Y$ can be expressed in a first-order Taylor series expansion about $V_{o}$, $\omega_{o}$ and $\eta_{o}[21]$-[22]. Taking into account that $s$ acts as a time differentiator, one obtains: 


$$
Y_{V} \Delta V(t)+Y_{\eta} \Delta \eta+Y_{\omega}\left(\Delta \omega_{q}+\dot{\phi}(t)-j \Delta \dot{V}(t) / V_{o}\right)=\sum_{k} \frac{I_{k}}{V_{o}} \exp j\left(-\phi(t)+\Delta \omega_{k, q} t\right)
$$

where $Y_{V}, Y_{\eta}$ and $Y_{\omega}$ are the derivatives of $Y$ with respect to $V, \eta$ and $\omega$, calculated at the freerunning steady-state oscillation $V_{o}, \omega_{o}, \eta_{o}$. These derivatives are obtained by means of the same AG used for the calculation of the free-running oscillation [10]. In that simulation, the AG operates at the unknown oscillation frequency $\omega_{0}$, with the amplitude $V_{\mathrm{o}}$, agreeing with the first harmonic of the node voltage. The AG must fulfill the oscillation condition $Y\left(V_{0}, \omega_{0}\right)=0$, which is solved through optimization in commercial HB software. Once the free-running solution has been determined, the derivatives of the admittance function are calculated through finite differences [13] (Fig. 1). The derivative $Y_{V}$ with respect to the amplitude is calculated by considering the increments $V_{o} \pm \Delta V$, while the frequency is kept at $\omega_{0}$. The frequency derivative $Y_{\omega}$ is calculated by considering the increments $\omega_{o} \pm \Delta \omega$, while the amplitude is kept at $V_{o}$. The derivative $Y_{\eta}$ with respect to the parameter is calculated by considering the increments $\eta_{o} \pm \Delta \eta$, while the AG amplitude and frequency are kept at $V_{o}, \omega_{o}$.

Solving (3) for $\Delta \omega_{q}+\dot{\phi}(t)$ one obtains:

$$
\Delta \omega_{q}+\dot{\phi}(t)=\frac{-\left|Y_{\eta}\right| \sin \alpha_{v \eta} \Delta \eta+\sum_{k} \frac{\left|I_{k}\right|}{V_{o}} \sin \left(-\phi(t)+\Delta \omega_{k, q} t+\phi_{k}-\alpha_{v}\right)}{\left|Y_{\omega}\right| \sin \alpha_{v \omega}}
$$

where $\left|I_{k}\right|$ and $\phi_{k}$ are the magnitude and phase of the input tones, $\alpha_{v_{\omega}}=\operatorname{ang}\left(Y_{\omega}\right)$ $\operatorname{ang}\left(Y_{V}\right), \alpha_{v \eta}=\operatorname{ang}\left(Y_{\eta}\right)-\operatorname{ang}\left(Y_{V}\right)$ and $\alpha_{v}=\operatorname{ang}\left(Y_{V}\right)$. Note that (4) describes the circuit response in both locked and unlocked conditions. When the oscillator is locked to $\omega_{q}$, the phase $\phi(t)$ in (4) can be represented in a Fourier series at $K-1$ frequencies $\Delta \omega_{1, q}, \ldots, \Delta \omega_{K, q}$, so one can express $\phi(t)=\phi_{o}+\Phi_{\text {mix }}(t)$, where $\phi_{o}$ is a constant value and $\Phi_{\text {mix }}(t)$ has zero average: $\left\langle\Phi_{\text {mix }}(t)=0>\right.$. Taking this into account, to detect the locking bands, one will introduce the expression $\phi(t)=\phi_{o}+\Phi_{m i x}(t)$ into (4) and perform an averaging:

$$
\begin{aligned}
& \left\langle\Delta \omega_{q}+\dot{\Phi}_{\text {mix }}(t)\right\rangle=\frac{\left|I_{q}\right|}{V_{o}} \frac{\left\langle\sin \left(-\phi_{o}-\Phi_{\text {mix }}(t)-\alpha_{v}\right)\right\rangle}{\left|Y_{\omega}\right| \sin \alpha_{v \omega}}-\frac{\left|Y_{\eta}\right| \sin \alpha_{v \eta}}{\left|Y_{\omega}\right| \sin \alpha_{v \omega}} \Delta \eta \\
& +\frac{\left\langle\sum_{k \neq q} \frac{\left|I_{k}\right|}{V_{o}} \sin \left(-\phi_{o}-\Phi_{\text {mix }}(t)+\Delta \omega_{k, q} t+\phi_{k}-\alpha_{v}\right)\right\rangle}{\left|Y_{\omega}\right| \sin \alpha_{v \omega}}
\end{aligned}
$$

Since $\Delta \omega_{q}$ is kept at a constant value, the average phase shift $\phi_{o}$ will vary with the tuning parameter $\Delta \eta$. Under small amplitudes $\left|I_{k}\right|, \Phi_{m i x}(t)$ can be neglected in the first term on the right hand side of (3). As the amplitudes $\left|I_{k}\right|$ decrease, the above equation approaches:

$$
\Delta \omega_{q}=\frac{\left|I_{q}\right|}{V_{o}} \frac{\sin \left(-\phi_{o}-\alpha_{v}\right)}{\left|Y_{\omega}\right| \sin \alpha_{v \omega}}-\frac{\left|Y_{\eta}\right| \sin \alpha_{v \eta}}{\left|Y_{\omega}\right| \sin \alpha_{v \omega}} \Delta \eta
$$

One can also solve (3) in terms of the increment of the voltage amplitude $\Delta V(t)$. Because the frequency reference is $\omega_{q}$, the voltage amplitude at this frequency is $V_{o}+\Delta V(t)$. Thus, the amplitude at the tone $\omega_{q}$ at which the oscillator is injection locked is enhanced by the selfoscillation and will be higher than that of the rest of spectral lines. Solving for the increment $\Delta V(t)$ and averaging, one obtains: 
$\langle\Delta V(t)\rangle=\frac{\frac{\left|I_{q}\right|}{V_{o}}\left\langle\sin \left(\alpha_{\omega}+\phi_{o}+\Phi_{m i x}(t)\right)\right\rangle}{\left|Y_{V}\right| \sin \alpha_{v \omega}}-\frac{\left|Y_{\eta}\right| \sin \alpha_{\eta \omega}}{\left|Y_{V}\right| \sin \alpha_{v \omega}} \Delta \eta+\frac{\left\langle\sum_{k} \frac{\left|I_{k}\right|}{V_{o}} \sin \left(\alpha_{\omega}-\phi_{o}-\Phi_{m i x}(t)+\phi_{k}-\Delta \omega_{k, q} t\right)\right\rangle}{\left|Y_{V}\right| \sin \alpha_{v \omega}}$

where $\alpha_{\omega}=\operatorname{ang}\left(Y_{\omega}\right), \alpha_{\eta \omega}=\operatorname{ang}\left(Y_{\omega}\right)-\operatorname{ang}\left(Y_{\eta}\right)$ and $\Delta \dot{V}(t)$ has been neglected. Taking into account the relationship between the average phase shift $\phi_{o}$ and $\Delta \eta$, the average of the amplitude increment $\langle\Delta V(t)\rangle$ should approach an ellipsoidal curve versus $\Delta \eta$ in the locking band. However, due to the presence of two turning points in the closed curve, only one section of this curve will be stable. Note that the turning points are local/global bifurcations [27]-[28] at which a quasiperiodic solution is generated from a zero frequency shift, in a manner analogous to the generation of a limit cycle in a saddle-node bifurcation [27]-[28].

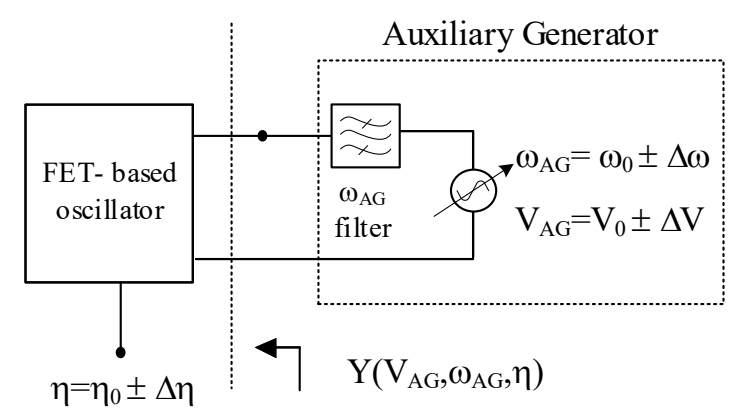

Fig. 1 Calculation of the derivatives of the oscillator nonlinear-admittance function through finite differences in harmonic balance, using an auxiliary generator.

\section{B) Application to a FET-based oscillator}

The above analysis has been applied to the voltage-controlled oscillator in Fig. 2. It is based on the transistor ATF-34143 and is tuned with the varactor SMV1235. It is able to oscillate in the frequency band $2.7 \mathrm{GHz}$ to $2.78 \mathrm{GHz}$. The input tones will be generated with the Agilent E4438C ESG Vector Signal Generator and are equally spaced by default. In order to generate as many tones as possible, the spacing $\Delta f=16 \mathrm{MHz}$ is chosen, enabling six input tones, between $2.7 \mathrm{GHz}$ and $2.78 \mathrm{GHz}$ (case (i) in Fig. 2). Initially, synchronization at $\omega_{q} /(2 \pi)=2.7 \mathrm{GHz}$ is assumed. First, the results obtained through the integration of (3) and with circuit-level envelope transient simulations [20] [using $\omega_{q}$ as the only fundamental frequency, as in (3)] have been compared. The spectrum obtained under injection-locked conditions for a multitone input signal in the two cases is shown in Fig. 3. As can be seen there is an excellent agreement that demonstrates the validity of (3). 


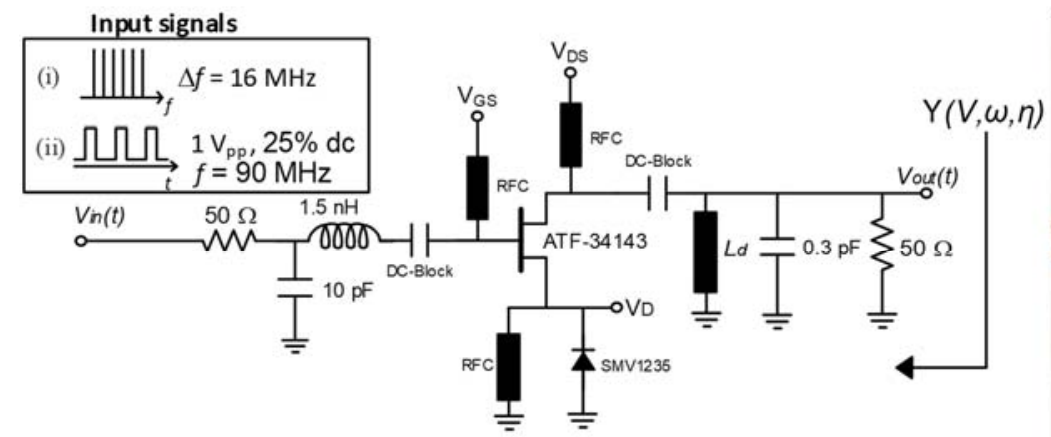

(a)

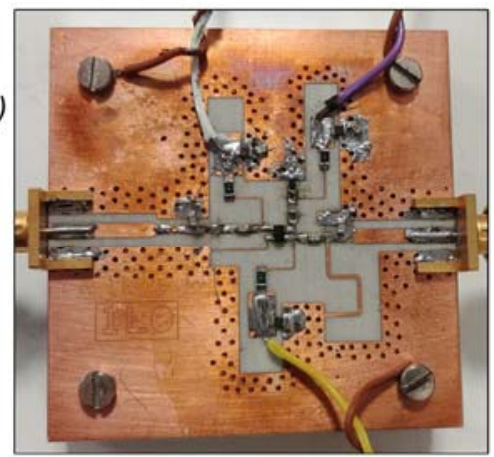

(b)

Fig. 2. FET-based (ATF-34143) oscillator at $f_{o}=2.7 \mathrm{GHz}$. The frequency is tuned with the varactor diode SMV1235. (a) Schematic, showing the two different kinds of input signal considered in this work: (i) six input tones equally spaced between $2.7 \mathrm{GHz}$ and $2.78 \mathrm{GHz}$ and (ii) a rectangular signal with $1 \mathrm{Vpp}, 25 \%$ duty cycle and frequency $90 \mathrm{MHz}$. The dc-blcok capacitance is suppressed in this second case. (b) Photograph.

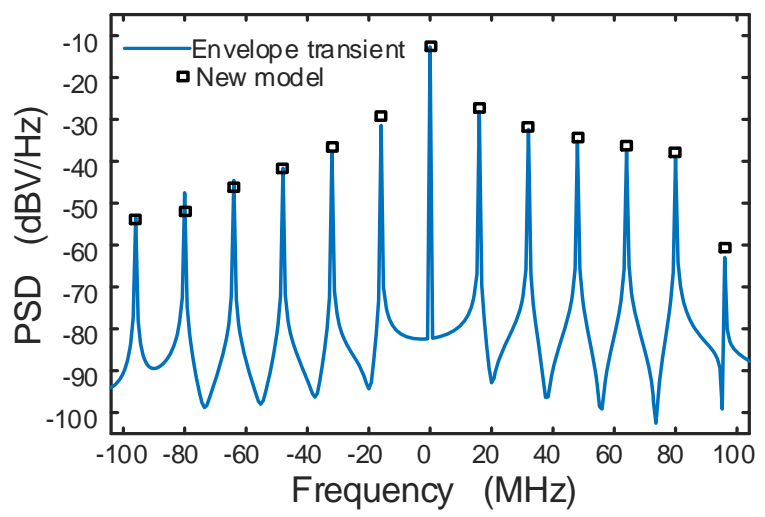

Fig. 3. Spectrum obtained through (3) and with envelope transient for the spectral-line power $P_{\text {in }}=-33 \mathrm{dBm}$ and frequency spacing $\Delta f=16 \mathrm{MHz}$.

Fig. 4(a) presents the phase shift $-\phi(t)$ (without averaging) for three different values of the tuning voltage $\eta=V_{D}$, within the synchronization band. In agreement with (4), after a transient, the phase exhibits a periodic variation about a constant value $-\phi_{o}$, which changes with the tuning voltage $V_{D}$. Note that the oscillator will get locked to a different input tone when varying $V_{D}$. To obtain the synchronization bandwidth about each input tone $\omega_{1}, \omega_{2}, \ldots, \omega_{K}$, one should change the reference frequency of the analysis. Otherwise there will be an additional ramp function $\left(\omega_{q}-\omega_{k}\right) t$ in $\Phi_{m i x}(t)$. The synchronization band is obtained representing the average of $-\phi(t)$ versus $V_{D}$, as shown in Fig. 4(b). This analysis of the synchronization band requires a sufficiently large time offset to ensure that the circuit is in steady state at each $V_{D}$ step. The apparent discontinuities in the diagram of Fig. 4(b) are because the fundamental frequency of the envelope-domain analysis, $\omega_{q}$, is, as stated, alternatively set to each of the input frequencies. The synchronization bands are easily distinguished (in solid line), since, in agreement with (6), $\langle-\phi(t)\rangle=-\phi_{o}$ varies continuously in each band, with an excursion of slightly less than $180^{\circ}$, corresponding to the stable solutions. This excursion is not centered about $\phi_{o}=0^{\circ}$ because $\alpha_{\nu}$ is 
different from zero. The three bands in Fig. 4(b) correspond to synchronization at $f_{1}=2.7 \mathrm{GHz}$, $f_{2}=2.716 \mathrm{GHz}$ and $f_{3}=2.732 \mathrm{GHz}$.

Fig. 5 presents the variation average amplitude $\langle\Delta V(t)\rangle$ in the same intervals of tuning voltage $V_{D}$ considered in Fig. 4. The same three locking bands are detected, distinguished by the ellipsoidal variation of $\langle\Delta V(t)\rangle$ versus $V_{D}$. Only the stable upper half of the ellipsoidal synchronization curves is obtained in the envelope-domain analysis. Note that the circuit becomes unlocked at the turning points of each curve.

The capability of the oscillator to discriminate a particular tone can be evaluated from the spectrum of the oscillation signal, without changing the fundamental frequency, kept at $\omega_{q}$. The frequencies the spectral line with larger output power and its neighboring spectral lines are identified and traced versus $V_{D}$ [Fig. 6(a)]. The circuit is synchronized where these frequency components remain constant when varying this parameter. Note that outside the synchronization band, mixing effects give rise to a significant variation of the dominant spectral component and its neighbors. In Fig. 6(b) the output power at the frequencies of the input tones is traced versus $V_{D}$, which allows evaluating the frequency-selection capability. The boundaries of the synchronization bands are easily detected by the fast variation of the spectrum frequencies, after the occurrence of each local/global bifurcation [10], [27]-[28]. The bandwidths slowly decrease when moving away from the oscillator free-running frequency $(2.7 \mathrm{GHz})$.

Fig. 7 presents the measurement results. Fig. 7(a) shows the six input tones, with $\Delta f=16$ MHz. Fig. 7(b) presents an unlocked spectrum for $V_{D}=0.28$ V. Fig. 7(c) and (d) present the spectrum for $V_{D}=0.8 \mathrm{~V}$ (synchronization to $f_{2}$ ), and $V_{D}=2.85 \mathrm{~V}$ (to $f_{5}$ ). We attribute the differences to inaccuracies and dispersion in the lumped-component models.
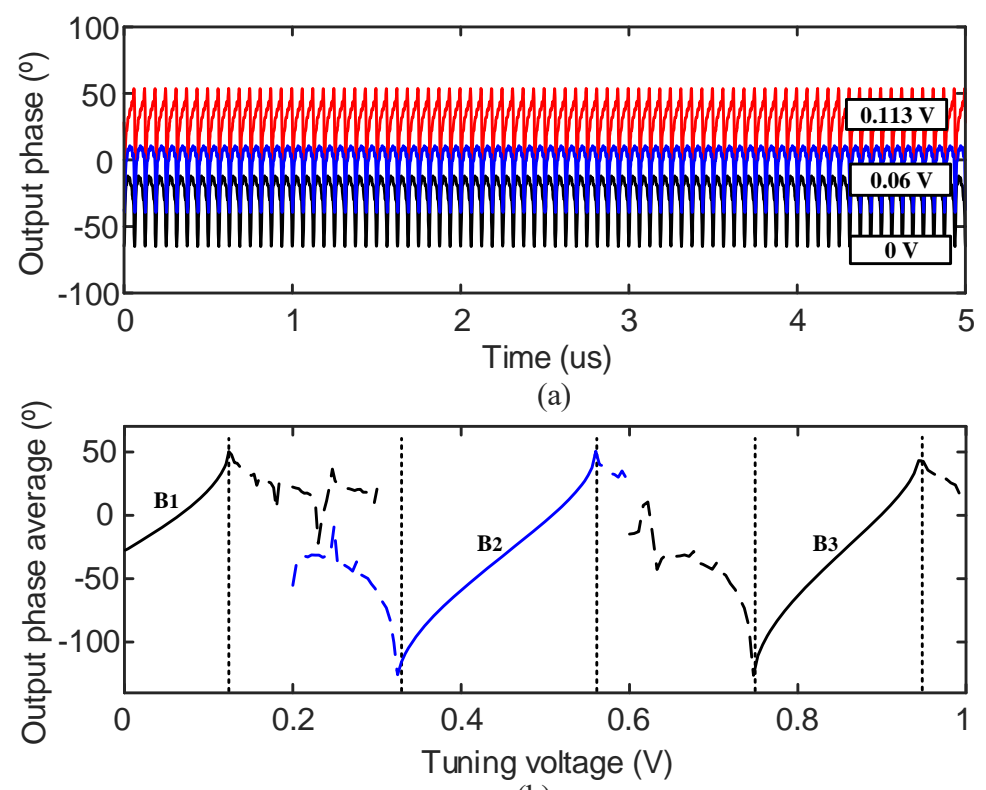

(b)

Fig. 4. Behavior under six input lines, between $2.7 \mathrm{GHz}$ and $2.78 \mathrm{GHz}$, with $\Delta f=16 \mathrm{MHz}$. (a) Time variation of the phase shift for three $V_{D}$ values, within the first synchronization band $\left(f_{1}=2.7 \mathrm{GHz}\right)$. (b) Average value of $-\phi(t)$ versus $V_{D}$. 


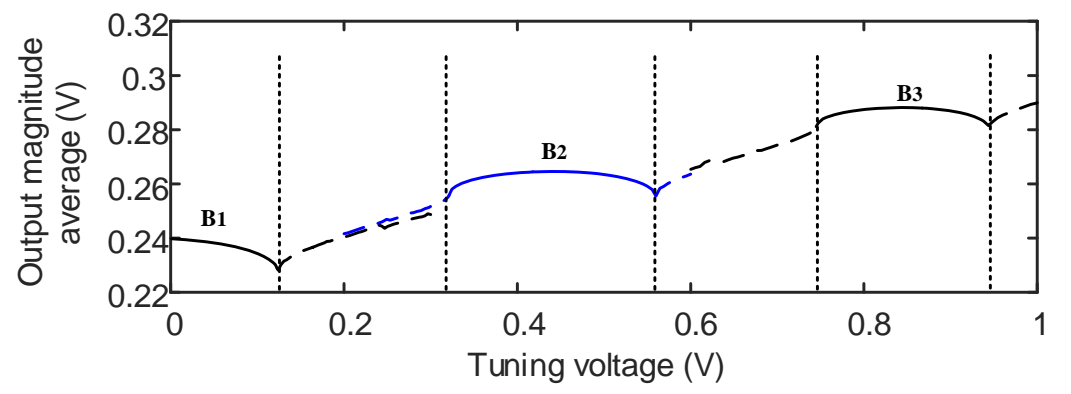

Fig. 5. Behavior under six input lines, between $2.7 \mathrm{GHz}$ and $2.78 \mathrm{GHz}$, with $\Delta f=16 \mathrm{MHz}$. Average value of $\Delta V(t)$ versus $V_{D}$, which approaches the upper half of an ellipse in each synchronization band.

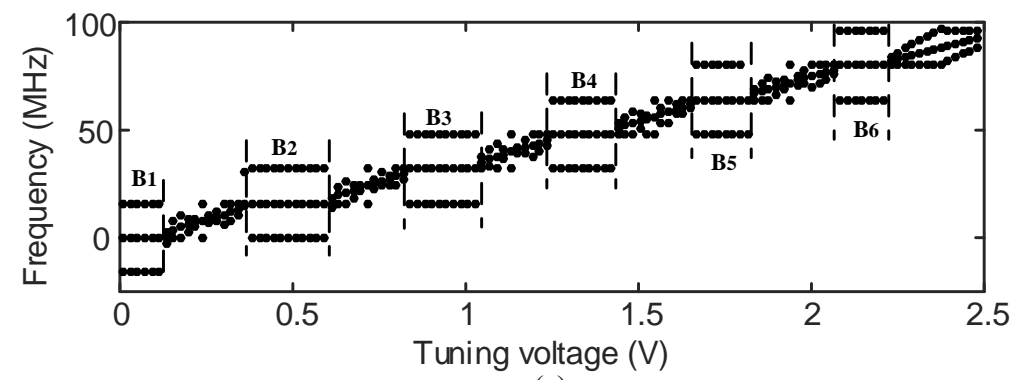

(a)

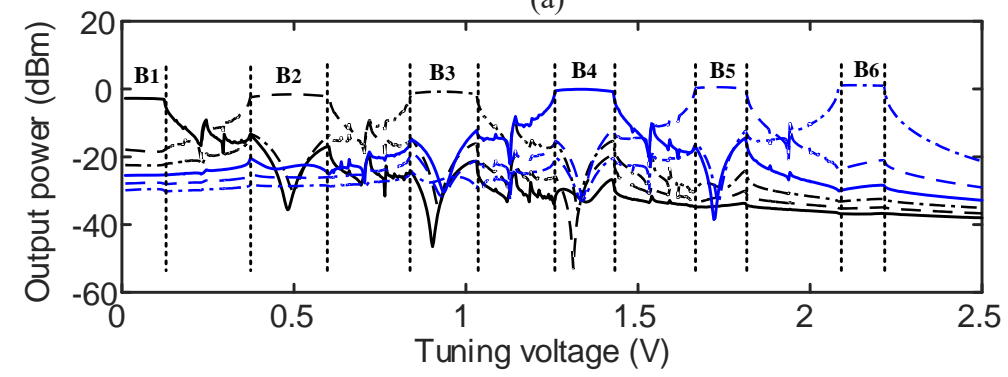

(b)

Fig. 6. Behavior under six input lines, between $2.7 \mathrm{GHz}$ and $2.78 \mathrm{GHz}$, with $\Delta f=16 \mathrm{MHz}$. Detection of synchronization bands from the analysis of the solution spectrum. (a) Frequencies of the spectral line with larger output power and its neighboring spectral lines, traced versus $V_{D}$.

(b) Output power at the frequencies of the input tones.
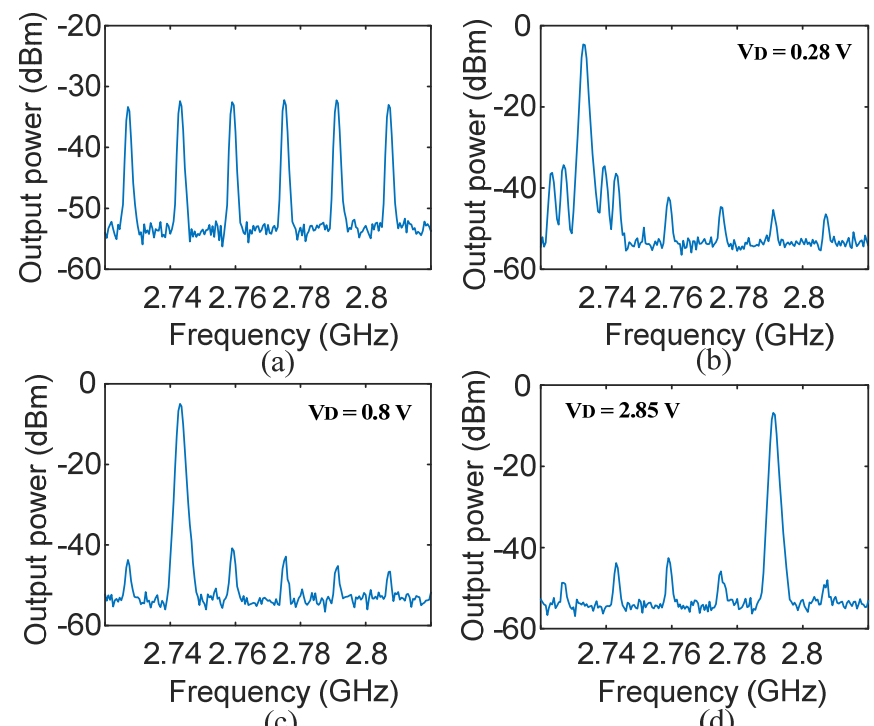

(c) 
Fig. 7. Measurement results. (a) Input tones. (b) Unlocked spectrum for $V_{D}=0.28 \mathrm{~V}$. (c) Synchronization to $f_{2}$ for $V_{D}=1.69 \mathrm{~V}$. (d) $f_{4}$ for $V_{D}=2.85 \mathrm{~V}$.

\section{Sub-harmonic injection locked operation}

In this section the case of a subharmonically injection-locked oscillator by an input signal of fundamental frequency $f_{\text {in }}$ will be considered. This analysis is more demanding than the one in Section II, since the subsynchronization is a nonlinear phenomenon and the relevant spectral lines, resulting from the input signal, plus frequency multiplication/mixing effects, cover the entire frequency bandwidth from DC to the oscillation frequency and its harmonic terms. When expressing the state variables in a Fourier basis at the fundamental frequency $f_{o}=N f_{i n}$, the system integration requires a small time step $\Delta t$, able to capture the large bandwidth going from dc to approximately $f_{o} / 2$. As a compromise, one can use a basis having $f_{o} / P$ as fundamental frequency, where the integer $P$ fulfils $P \leq N$. The analysis can be carried out representing the circuit variables in a Fourier series $\sum_{k} X_{k}(t) \exp \left(j k \omega_{o} / P\right)$, where $P$ is a positive integer.

A schematic representation of the Fourier basis is shown in Fig. 8. For $P=N$, the harmonic components $\bar{X}_{k}(t)$ should take constant values in the locked steady-state. Despite the high number of harmonic terms, the complexity will be smaller than in a HB simulation. This is because the oscillation is easily initialized by just connecting an auxiliary generator at the oscillation frequency at the initial time only [20], [29]. The amplitude of this generator can be the same obtained in free-running conditions, $V_{o}$. For $P<N$, there will be several spectral lines of the injection-locked solution about each harmonic term. The integration time step of the envelopetransient system must be small enough to properly sample the frequency components located in the bandwidth $\left[-\omega_{o} /(2 P), \omega_{o} /(2 P)\right]$ associated with $\bar{X}_{k}(t)$, at the central frequency $k \omega_{o} / P$. Although the allocation of the harmonic components of $\omega_{o} / N$ is not unique, all the possible arrangements of these components will fulfil the envelope-domain system at each time value, producing the same time domain signal when gathered in the Fourier series.

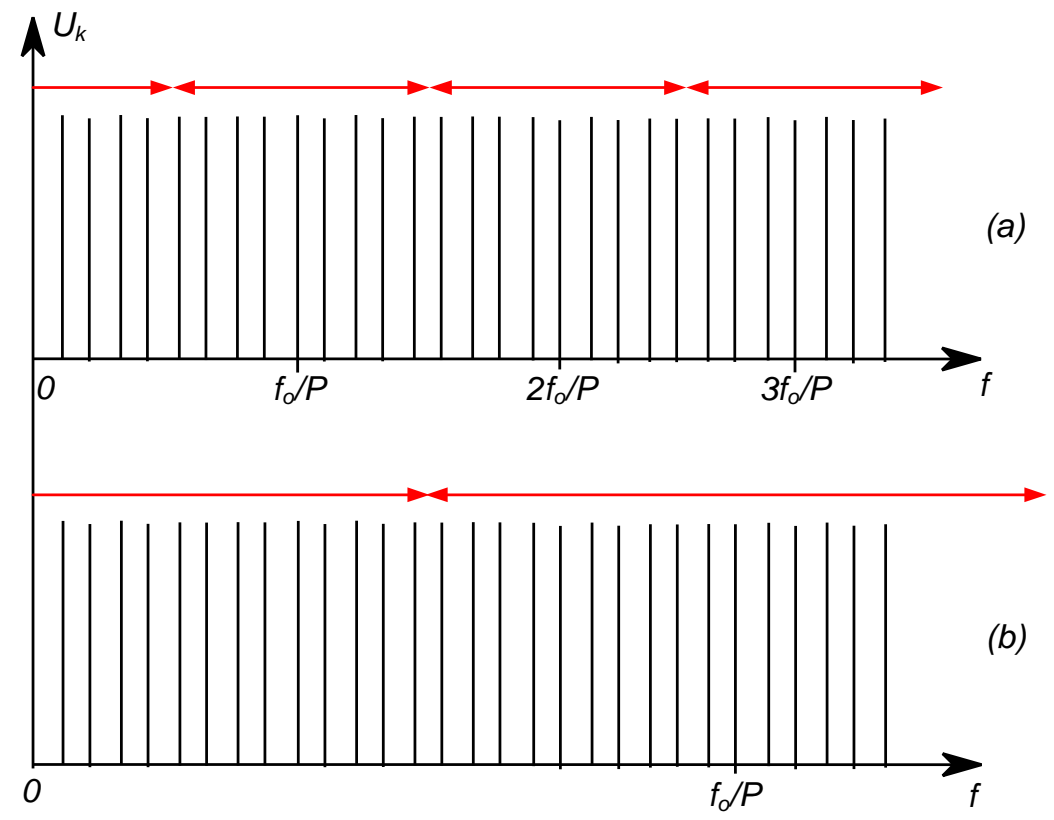

Fig. 8. Envelope-domain analysis of the high-order subharmonic injection-locked oscillator. (a) and (b) Different choices of the fundamental frequency $f_{o} / P$ in the Fourier-series representation of the circuit variables. 
For better insight, a reduced-order formulation from a single observation node will be considered. Setting the fundamental frequency to $\omega_{o} / P$, one can express the outer-tier envelopetransient system is:

$$
\begin{aligned}
& G\left(V_{0}(t), \tilde{V}_{1}(t), \ldots, \tilde{V}_{P}(t), s / j\right) V_{o}(t)=\sum_{k=1}^{B / 2} I_{k} \exp j\left(\omega_{k} t\right), \\
& Y_{1}\left(V_{0}(t), \tilde{V}_{1}(t), \ldots, \tilde{V}_{P}(t), \omega_{o} / P+s / j\right) \tilde{V}_{1}(t)= \\
& \sum_{k=1+B / 2}^{B+B / 2} I_{k} \exp j\left(\Delta \omega_{1, k} t\right), \Delta \omega_{1, k}=\omega_{k}-\omega_{o} / P \\
& \vdots \\
& Y_{p}\left(V_{0}(t), \tilde{V}_{1}(t), \ldots, \tilde{V}_{P}(t), p \omega_{o} / P+s / j\right) \tilde{V}_{p}(t)= \\
& \quad \sum_{k=1+(p-1) B+B / 2}^{1+p B+B / 2} I_{k} \exp j\left(\Delta \omega_{p, k} t\right), \Delta \omega_{p, k}=\omega_{k}-p \omega_{o} / P \\
& \vdots Y_{P}\left(V_{0}(t), \tilde{V}_{1}(t), \ldots, \tilde{V}_{P}(t), \omega_{o}+s / j\right) \tilde{V}_{P}(t)= \\
& \sum_{k=1+(P-1) B+B / 2}^{M} I_{k} \exp j\left(\Delta \omega_{P, k} t\right), \Delta \omega_{P, k}=\omega_{k}-\omega_{o}
\end{aligned}
$$

where $\tilde{V}_{p}(t)$ indicates phasors. In (8), the integer $B / 2$ is the number of spectral lines at each side of the $p$-th subharmonic component, $p f_{\mathrm{o}} / P$, where $p=1, \ldots, P$. Thus, the total number of spectral lines is given by $M=B / 2+P B$. The frequency-domain models of the distributed components must be valid in the whole bandwidth of each harmonic component. For a higher $P$, the integration time step can, in principle, be increased as $P \Delta t$. Note that the equation at each harmonic $p$ is analogous to the one in (2). The phasors $\tilde{V}_{p}(t)$, with the phases $\phi_{p}(t)=\phi_{o, p}+\Phi_{m i x, p}(t)$, will exhibit a slower time variation for a higher $P$. In the limit situation $P=$ $N$, the phasors $\tilde{V}_{p}$ will be constant in the synchronization band. For $P<N$, the components $\bar{X}_{k}(t)$ will be periodic in the locked steady state, with this time variation being due to the neighbouring equally-spaced spectral lines.

Unlike the envelope-domain equations (3) to (7), based on a linearization of the admittance function about the free-running solution, (8) will not be numerically resolved. In (3) to (7) the oscillator behaves linearly with respect to the $K$ input sources, with frequencies located about the free-running one. In contrast, in (8) the oscillator will (generally) behave nonlinearly with respect to the input source at a much lower frequency. The input source plus the device nonlinearity must be able to generate a harmonic signal at the oscillation frequency, able to injection lock the oscillation. In principle, the admittance at each harmonic frequency $p$ depends on all the harmonic voltages at the observation node, so the extraction of these admittance functions is virtually impossibe. The purpose of equation (8) is to illustrate the various possible ways to partition the spectrum when analyzing the high-order subharmonic injection-locked oscillator with the envelope-transient method and their impact on the integration time step.

To obtain the sub-harmonic injection locking, a rectangular signal, at a frequency $f_{o} / N$, is injected into the high-frequency oscillator, as shown in Fig. 2, case (ii). The rectangular signal contains a high number of harmonic terms of $f_{o} / N$, which facilitates the subharmonic injection locking. Note that this is different from the synthesis method in [1]-[3]. In those works, a large multiplication factor is achieved by shaping the input sinusoidal signal (at low frequency), into a square signal. The shaped signal periodically switches on and off a high-frequency oscillator, 
suitably tuned to maximize the power at the desired harmonic terms. The resulting signal is used to injection lock a second oscillator, which enables the selection of one or another spectral line.

Here a rectangular signal is introduced into the oscillator of Fig. 2. The signal has a frequency $90 \mathrm{MHz}$, amplitude $1 \mathrm{Vpp}$, and duty-cycle of $25 \%$. One must note that although the oscillation frequency of this prototype $(2.7 \mathrm{GHz})$ is lower than the one in [1]-[3], the frequency ratio is the same $N=30$, so the analysis complexity must be similar. The circuit has been analysed with circuit-level envelope transient, following the criteria in (8), and using an auxiliary generator at $f_{o}$, connected to the circuit at the initial time only to initialize the oscillation, as shown in [20]. Fig. 9 presents the spectrum at $f_{\text {out }}=30 \times 90 \mathrm{MHz}$ for $P=1,4,6$, which predicts a synchronized behaviour. Only the central spectral lines can agree since for $P>1$ part of the spectrum corresponds to terms $P-1$ and $P+1$. For $P=1$ the integration time step is $0.05 \mathrm{ns,}$, whereas for $P=$ 6 it is $0.3 \mathrm{~ns}$. The analysis has been validated through an independent HB analysis (superimposed). This costly HB analysis has been carried out providing the amplitude and phase of the central spectral line, obtained with envelope transient, to an auxiliary generator at $f_{\text {out }}=30 \times 90 \mathrm{MHz}$. With $P=6$, the time variation is more regular, since less spectral lines contribute to this variation at each harmonic term. Thus, the synchronization bands can be determined with higher accuracy.

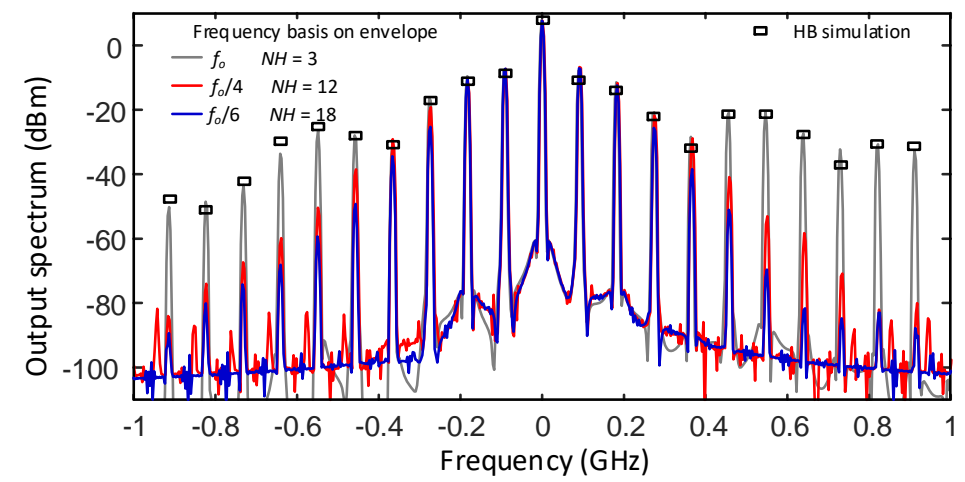

Fig. 9. Injection locking by a pulsed signal at $90 \mathrm{MHz}$. Output spectrum at $f_{\text {out }}=30 \times 90 \mathrm{MHz}$ when considering $P=1,4,6$. HB results are superimposed.

In this particular case $(N=30)$, it was possible to perform a HB simulation. Fig. 10 presents the solution of the circuit in Fig. 2 when driven with a rectangular waveform of $V_{\text {peak }}=1 \mathrm{~V}$, frequency $f_{\text {in }}=90 \mathrm{MHz}$ and duty cycle $25 \%$. The spectrum in Fig. 10(a) was obtained with default HB. The power of the spectral lines about the oscillation frequency $(2.7 \mathrm{GHz})$ is well below that of the original free-running oscillation $(7.75 \mathrm{dBm})$. Fig. $10(\mathrm{~b})$ presents the spectrum obtained with an AG, operating at $N f_{\text {in }}$, through the optimization of its amplitude $V$ and phase $\phi$, in order to fulfill: $Y(V, \phi)=0$. As can be seen, there is a spectral line with a much higher output power, which corresponds to the injection-locked oscillation. If the solution obtained through AG optimization is storaged in an ASCII file and using it in default $\mathrm{HB}$ as an initial condition, the resulting spectrum is overlapped, which validates the accuracy of the AG-based procedure.By sweeping the phase $\phi$ and optimizing $V$ and $V_{D}$ in order to fulfill: $Y\left(V, V_{D}\right)=0$, it was possible to trace the synchronization curve versus $V_{D}$ through AG optimization in HB [10], which took approximately 3 hours. The resulting closed synchronization curve (bounded by the two turning points corresponding to local/global bifurcations) is shown in Fig. 11(a). Experimental points are superimposed. The envelope-domain analysis is computationally more efficient. Fig. 11(b) and Fig. 11(c) present the analysis of the synchronization band with the averaging method, when considering $P=6$. Fig. 11(b) presents the variation of the average value of $P$-th harmonic component of the output voltage, $\left\langle\phi_{P}(t)\right\rangle=\phi_{o, P}$, versus $V_{D}$. which enables the detection of the locking band. Fig. 11(c) 
presents the result of averaging the amplitude of $P$-th harmonic component of the output voltage, denoted as $\left\langle V_{P}(t)\right\rangle=V_{o, P}$. The envelope-domain integration only provides the upper (stable) section of the synchronization curve. There is an excellent agreement with the HB predictions, though the computation time is four times shorter. Note that the comparison with HB should be performed in terms of the tuning-voltage interval with locked operation. This is determined by the two turning points of the ellipsoidal curve, in the case of HB simulations, and the slope discontinuity, in the case of envelope-transient simulations. As can be seen, the locked-operation interval is the same with the two simulation methods.

Note that it was not possible to obtain any HB convergence when changing $V_{D}$ in order to select a different spectral line, for instance $N+1$ and $N+2$. This could be easily achieved with the envelope-transient method, as shown in Fig. 12(a) to (c), where three different spectral lines, corresponding to $N=30, N+1$ and $N+2$, are selected by varying $V_{D}$. Finally, the output of the subsynchronized oscillator has been connected to an analogous voltage-controlled oscillator to increase the frequency selectivity. To initialize the two individual oscillations an AG is connected to each circuit at the initial time only. The simulated spectra are shown in Fig. 13(a) and (b). The ratio between the selected spectral line and the highest-power neighbouring line is in the order of $30 \mathrm{~dB}$. Fig. 13(b) presents the experimental results. In a manner similar to Fig. 7, there is a deviation in the tuning voltages, attributed to the device models.
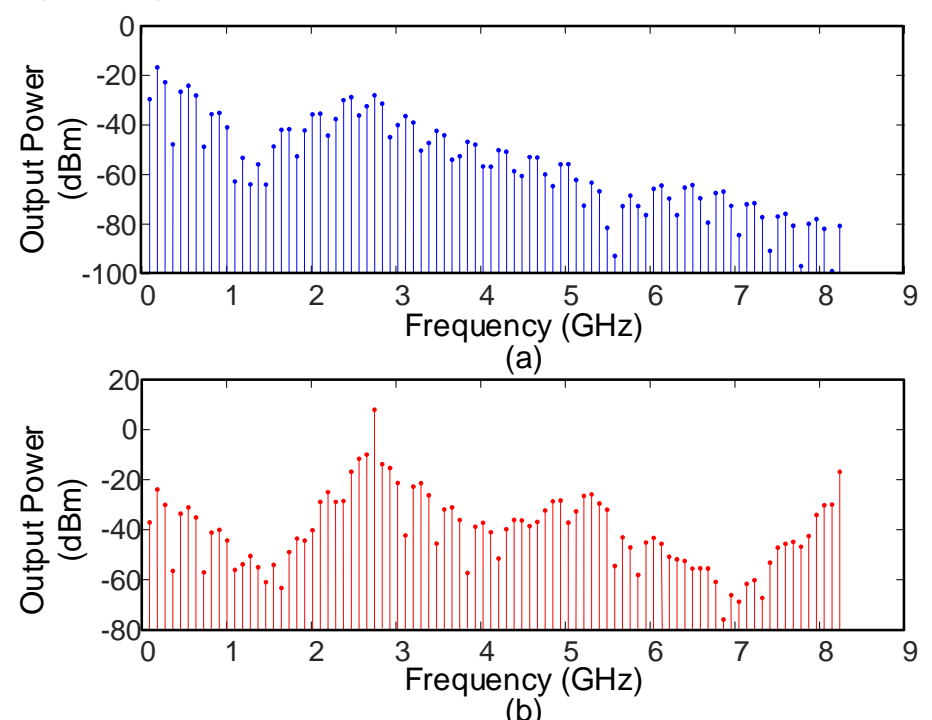

Fig. 10. HB analysis of the circuit in Fig. 2 under a rectangular input signal of $V_{\text {peak }}=1 \mathrm{~V}$, frequency $90 \mathrm{MHz}$ and duty cycle $25 \%$. (a) Without AG. The oscillation is not excited. (b) With an $\mathrm{AG}$, after fulfilment of the optimization condition $Y(V, \phi)=0$. The injection-locked oscillation is clearly visible. When storing the solution obtained through AG optimization in an

ASCII file and using it in default HB as an initial condition, the resulting spectrum is overlapped, which validates the accuracy of the AG-based procedure 

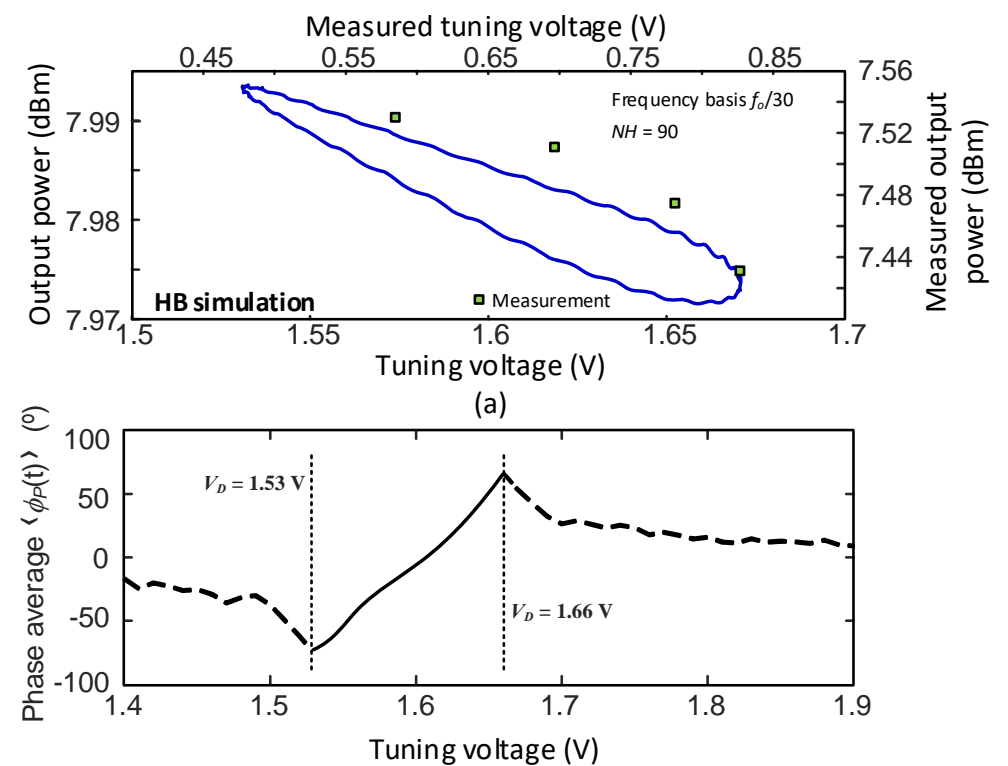

(b)

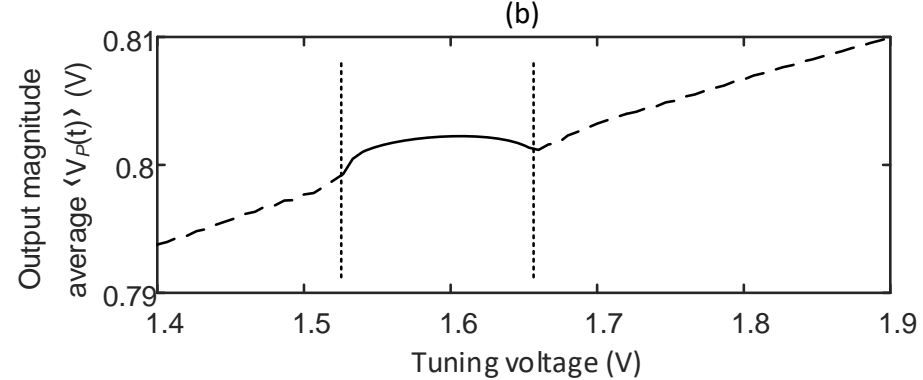

(c)

Fig. 11. Locking band at $N=30$. (a) Synchronization curve, versus $V_{D}$, obtained with HB.

Experimental points are superimposed. (b) Envelope-domain analysis with $P=6$. Evolution of the phase average $\left\langle\phi_{P}(t)\right\rangle=\phi_{o, P}$ versus $V_{D}$. (c) Evolution of the amplitude average $\left\langle V_{P}(t)\right\rangle=V_{o, P}$ versus $V_{D}$. 


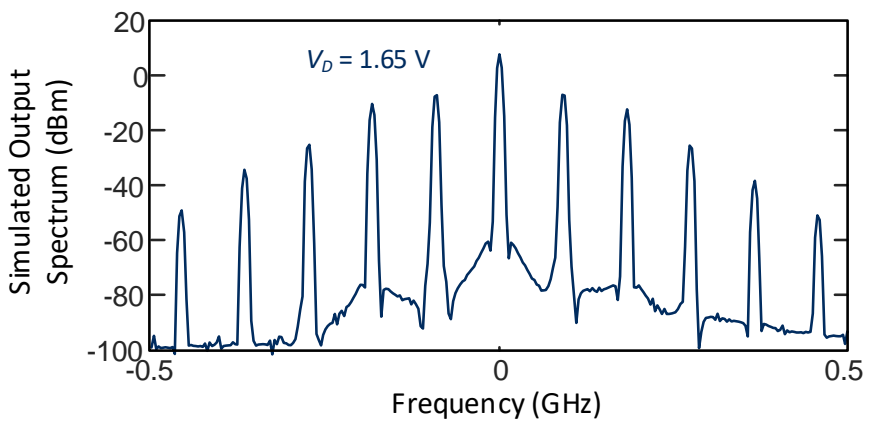

(a)
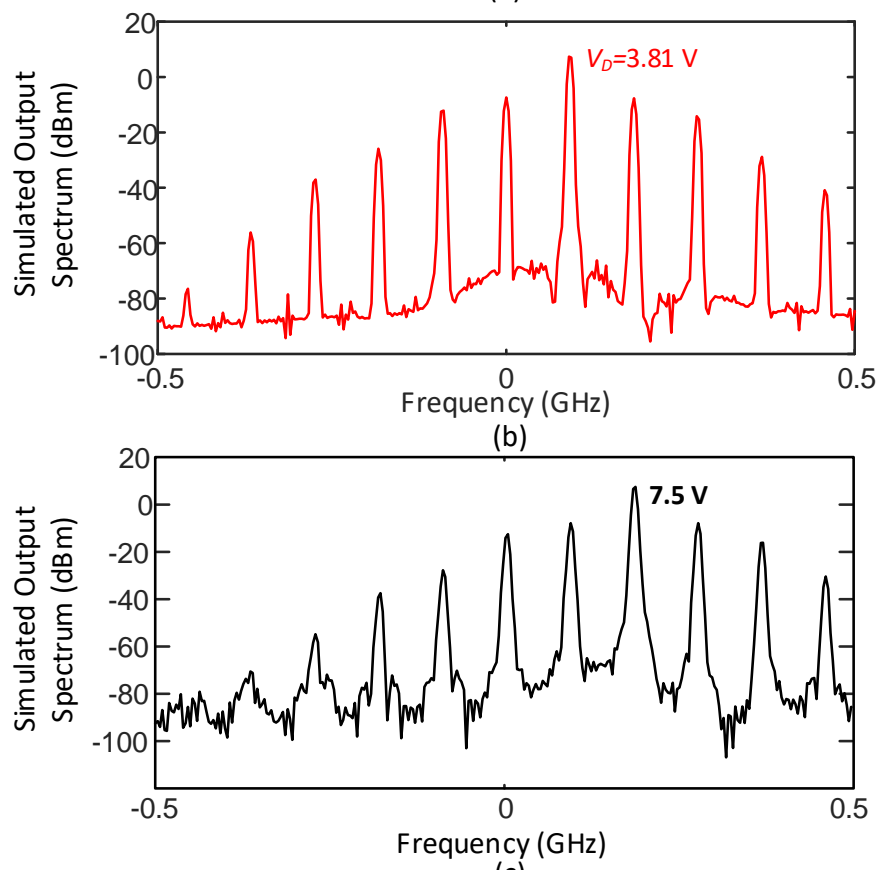

(c)

Fig. 12. Oscillator locking to different spectral lines of the input source by tuning $V_{D}$. (a) Locking to $f_{1}=2.7 \mathrm{GHz}(N=30)$. (b) Locking to $f_{2}=2.79 \mathrm{GHz}(N+1)$. (c) Locking to $f_{3}=2.88$ $\mathrm{GHz}(N+2)$. 

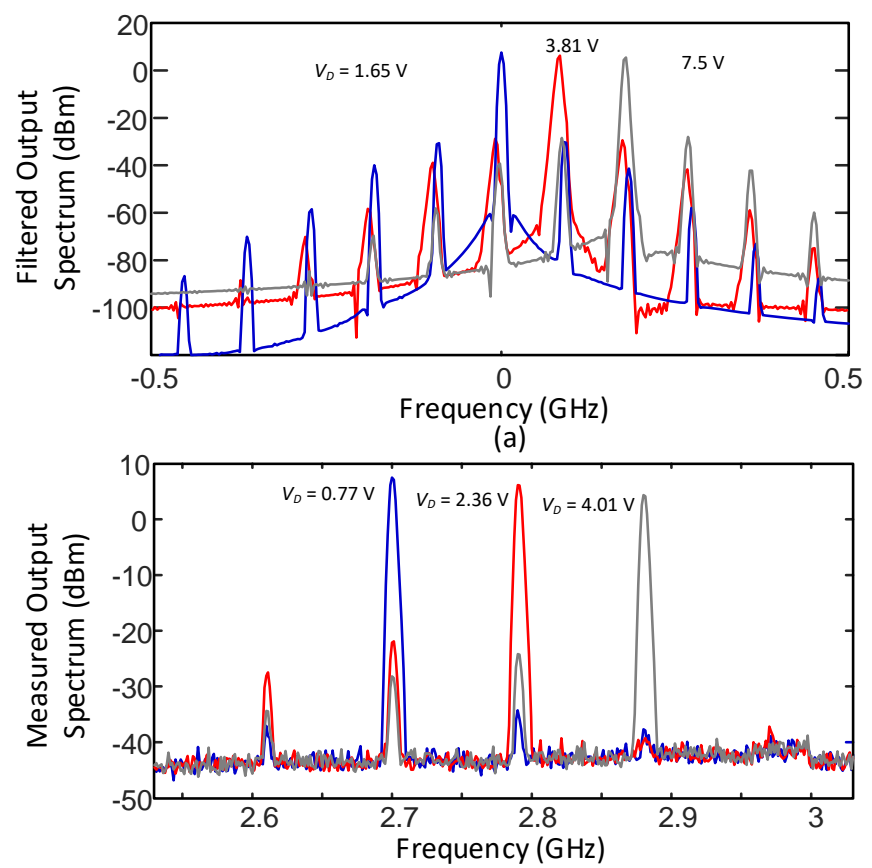

(b)

Fig. 13 Increase of the frequency selectivity through the use of a second oscillator. (a) Simulations. (b) Measurements.

\section{Phase noise}

The phase-noise analysis of the subharmonic injection-locked oscillator at a high order $N$ is challenging. This is because, in most cases, the only way to obtain the steady-state solution is through an envelope-domain analysis using the criteria discussed in Section III. Then, one faces the problem of two different time scales, in addition to the harmonic frequencies $k f_{o} / P$ considered in the Fourier-series representation of the circuit variables. One of the additional time scales is slow, because it is associated with the noise perturbations. The other one is faster, and depends on the $P$ value selected in (6), which determines the bandwidth about each spectral line in the Fourierseries representation of the circuit variables. Two cases will be considered here, $P=N$, for which the harmonic components of the Fourier series considered in (6) take constant values, and $P<N$ for which the harmonic components are time varying. The case $P=N$ will be addressed by means of a two-tier HB analysis, with the admittance function of the injection-locked oscillator at $f_{o}=N f_{\text {in }}$ constituting the outer tier. This will allow an understanding of the phase-noise response of the high-order subharmonic injection-locked oscillator to the noise sources. In the case $P<N$ the key parameters determining the phase-noise response will be identified from envelope-domain simulations through a numerical procedure.

\section{A) Case $P=N$}

For $P=N$ the subharmonic injection-locked oscillator will be described with an outer-tier admittance function $Y$ extracted from HB simulations. This admittance function is similar to the one considered in Section II. However, the circuit is now in injection-locked conditions with respect to the subharmonic source, at the high subharmonic order $N$. Thus, the function $Y$ depends on the observation node amplitude $V$ and phase $\phi$, since the oscillation frequency is determined by the input source frequency and fulfils $f_{o}=N f_{i n}$. Thus, the synchronized solution will fulfil the following two-tier equation system: 


$$
\begin{aligned}
& Y\left(V_{s}, \phi_{s}\right)=0 \\
& \bar{H}\left(\bar{X}^{\prime}, V_{s}, \phi_{s}\right)=0 \\
& \omega_{o}=N \omega_{i n}
\end{aligned}
$$

where $\bar{H}$ represents the harmonic balance system that constitutes the inner tier, $V_{s}$ and $\omega_{s}$ are, respectively, the amplitude and phase of the steady-state solution and the vector $\bar{X}^{\prime}$ contains all the state variables except the voltage at the observation node $V e^{j \phi}$ and its complex conjugate. The above system can be solved through the optimization of $V$ and $\phi$ in order to fulfil the goal $Y=0$

For the noise analysis, two different contributions will be considered: the noise from the input source and the circuit own noise sources. Regarding the input source, the phase noise will be much larger than the amplitude noise, so the latter will be neglected. On the other hand, the circuit noise will be modelled with an equivalent noise-current generator at the observation node $i_{n}(t)$. The spectral density of this equivalent noise-current source $\left|I_{N}(\Omega)\right|^{2}$ is fitted with the oscillator in free-running conditions, in order to obtain the same phase-noise spectral density as with the entire set of circuit noise sources. The fitting is carried out in circuit-level HB, using the conversion-matrix approach [30]-[32]. The result is shown in Fig. 14. Note that in injection-locked conditions, one can expect the oscillator to follow the phase noise of the input source (usually growing as $\Omega^{3}$ when approaching this carrier) up to a certain (relatively high) offset frequency, above which the impact of flicker noise will usually be negligible. Thus, the fitting can be limited to the effect of the white noise sources.

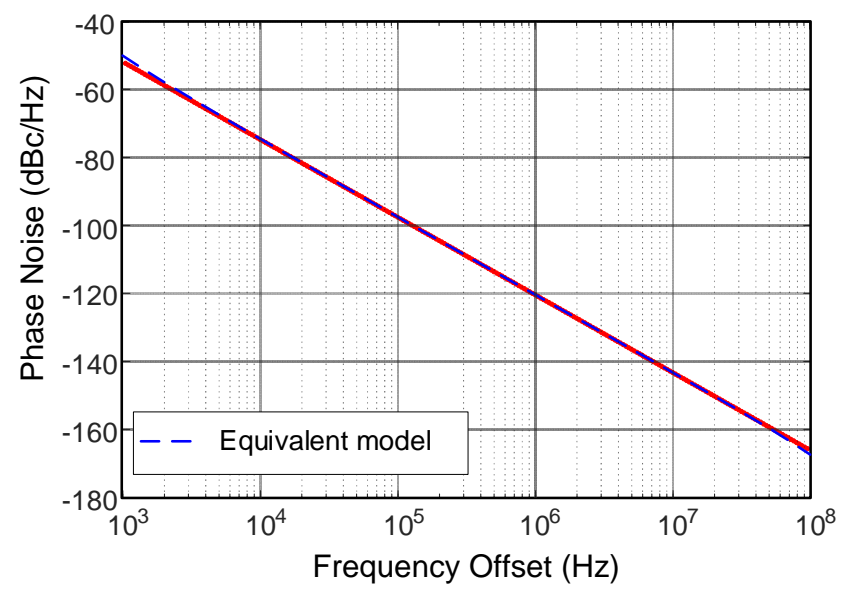

Fig. 14. Fitting the spectral density of the original free-running oscillator with a single equivalent current source of spectral density $\left|I_{N}(\Omega)\right|^{2}$. The fitting is carried out in circuit-level HB, using the conversion-matrix approach.

The phase noise contribution of the input source (with arbitrary waveform) will be obtained from the jitter $\tau(t)$ of this source [33]-[34]. One must take into account that a time translation $\tau$ of the input signal gives rise to an identical time translation of the oscillator solution, which does not affect the value the admittance function $Y\left(V_{s}, \phi_{s}\right)$, depending only on the phase shift with respect to the input source. However, the time translation will modify the absolute node phase, which is increased as $\phi+N \omega_{\text {in }} \tau$. Thus, the jitter of the input source will give rise to the phase perturbation 
$N \psi(t)=N \omega_{i n} \tau(t)$ at the oscillation frequency. Because there are also noise contributions coming from the oscillator circuit [modelled with the equivalent source $i_{n}(t)$ ], the total phase perturbation of the voltage at the observation node will be $\Delta \phi_{T}(t)=N \omega_{i n} \tau(t)+\Delta \phi(t)$ where $\Delta \phi(t)$ is a small increment with respect to the steady-state phase $\phi_{o}$ due to the noise perturbations. On the other hand, the node amplitude, under the influence of $\tau(t)$ and $i_{n}(t)$ becomes $V_{s}+\Delta V(t)$.

In the presence of the noise perturbations the outer-tier system at $N \omega_{\text {in }}$ can be obtained by performing a first-order Taylor series expansion of the total node admittance $Y$ about the particular steady-state synchronized solution, $V_{s}, \phi_{s}$ and $\omega_{s}$, which provides:

$$
\left[Y_{V} \Delta V(t)+Y_{\phi} \Delta \phi(t)-j Y_{\omega} s\right]\left(V_{s}+\Delta V(t)\right) e^{j\left(N \omega_{i n} \tau(t)+\Delta \phi(t)\right)}=I_{N}(t)
$$

where $s$ acts like a time differentiator and the subindexes stand for derivatives of $Y$ with respect to the corresponding variables $V, \phi$ and $\omega$ and $I_{N}(t)$ is the envelope of the current-noise source $i_{n}(t)$ about $N \omega_{s}$. After the time differentiation, one obtains the following equation:

$$
Y_{V} \Delta V(t)+Y_{\omega}\left(\left(N \omega_{i n} \dot{\tau}(t)+\Delta \dot{\phi}(t)\right)-j \frac{\Delta \dot{V}(t)}{V_{s}+\Delta V(t)}\right)+Y_{\phi} \Delta \phi(t)=\frac{I_{N}(t)}{V_{s}}
$$

where the subindexes indicate the quantity with respect to which the admittance function is differentiated. The derivatives $Y_{V}, Y_{\omega}, Y_{\phi}$ are calculated at the steady-state synchronized solution through finite differences in HB, following the procedure described in [13], [21].

Next, the complex equation (8) is split into real and imaginary parts and the Fourier transform is applied in the slowly-varying time scale of the noise perturbations, with associated frequency $\Omega$. Solving for $\Delta \phi_{T}(\Omega)=\Delta \phi(\Omega)+N \psi(\Omega)$ and multiplying by the adjoint, the phasenoise spectral density [21] is given by:

$$
\left|\Delta \phi_{T}(\Omega)\right|^{2}=\frac{\left|Y_{\mathrm{v}} \times Y_{\phi}\right|^{2} N^{2}|\psi(\Omega)|^{2}+2\left|Y_{V}\right|^{2} \frac{\left|I_{N}\right|^{2}}{V_{s}^{2}}}{\left|Y_{V} \times Y_{\phi}\right|^{2}+\left|Y_{V} \times Y_{\omega}\right|^{2} \Omega^{2}}=\frac{\left|Y_{\phi}\right|^{2} \sin ^{2} \alpha_{V \phi} N^{2}|\psi(\Omega)|^{2}+2 \frac{\left|I_{N}\right|^{2}}{V_{s}^{2}}}{\left|Y_{\phi}\right|^{2} \sin ^{2} \alpha_{V \phi}+\left|Y_{\omega}\right|^{2} \sin ^{2} \alpha_{V \omega} \Omega^{2}}
$$

where higher order terms in $\Omega$ have been neglected and the complex-number products of the form $a \times b$ are real and are defined as: $a \times b=\operatorname{Re}(a) \operatorname{Im}(b)-\operatorname{Re}(b) \operatorname{Im}(a)=|a||b| \sin (\angle b-\angle a)$. In turn, the angles in the third term are defined as $\alpha_{a b}=\angle b-\angle a$. To derive the above expression it has been taken into account that the real and imaginary parts of the equivalent noise source $\bar{I}_{N}$ are uncorrelated and the input noise is uncorrelated with the oscillator noise. Dividing the numerator and denominator by $\left|Y_{\mathrm{V}} \times Y_{\phi}\right|^{2}$, one obtains:

$$
\left|\Delta \phi_{T}(\Omega)\right|^{2}=\frac{N^{2}|\psi(\Omega)|^{2}+\frac{1}{\left|Y_{\phi}\right|^{2} \sin ^{2} \alpha_{V \phi}} 2 \frac{\left|I_{N}\right|^{2}}{V_{s}^{2}}}{1+\frac{\left|Y_{\omega}\right|^{2} \sin ^{2} \alpha_{V \omega}}{\left|Y_{\phi}\right|^{2} \sin ^{2} \alpha_{V \phi}} \Omega^{2}}
$$

which can be rewritten as: 


$$
\left|\Delta \phi_{T}(\Omega)\right|^{2}=\frac{N^{2}|\psi(\Omega)|^{2}+A_{p} \frac{\left|I_{N}\right|^{2}}{V_{s}^{2}}}{1+\frac{\Omega^{2}}{\omega_{3 d B}^{2}}}
$$

where the following parameters have been introduced:

$$
A_{p}=\frac{2}{\left|Y_{\phi}\right|^{2} \sin ^{2} \alpha_{V \phi}}, \quad \omega_{3 d B}=\frac{\left|Y_{\phi}\right| \sin \left(\alpha_{V \phi}\right)}{\left|Y_{\omega}\right|\left(\sin \alpha_{V \omega}\right)}
$$

As gathered from (14), the injection-locked oscillator exhibits a low-pass response with respect to the input phase noise and its own noise sources, with a $3 \mathrm{~dB}$ cut-off frequency $\omega_{3 d B}$. As shown in (15), the corner frequency $\omega_{3 d B}$ will be, in principle, smaller for a higher quality factor of the oscillator (larger $\left|Y_{\omega}\right|$ ) and larger for a higher $\left|Y_{\phi}\right|$, implying a higher sensitivity to the input source and thus a larger locking range. Note, however, that the angles $\alpha_{V \phi}, \alpha_{V \omega}$ can be very relevant.

For small offset frequency, the phase noise follows $N^{2}|\psi(\Omega)|^{2}$, as derived from the jitter $\tau(t)$ of the input signal. The effect of the circuit noise sources is determined by $A_{p}\left|I_{N}\right|^{2} / V_{s}^{2}$. If $A_{p}\left|I_{N}\right|^{2} / V_{s}^{2}>N^{2}|\psi(\Omega)|^{2}$ from a certain $\Omega<\Omega_{3 \mathrm{~B}}$, before decaying $-20 \mathrm{~dB} / \mathrm{dec}$.

The predictions of the semi-analytical expression (14) have been compared with a phasenoise analysis of the subharmonic injection-locked oscillator at $N=30$, performed in $\mathrm{HB}$, with the conversion-matrix approach [30]-[32]. The results of this comparison, in the presence of the equivalent white-noise source $i_{n}(t)$, are shown in Fig. 15. The curves providing the phase-noise spectral density obtained with the two methods are overlapped. Remember that the spectral density of the white-noise source $\left|I_{N}\right|^{2}$ was fitted in the standalone free-running oscillator. The corner frequency is $f_{3 \mathrm{~dB}} \cong 1.5 \mathrm{MHz}$

For the prediction of the whole spectrum, the phase noise of the rectangular input source at the fundamental frequency $(90 \mathrm{MHz})$ has been experimentally characterized, using the R\&S FSWP8 - Phase Noise Analyzer, and introduced in (14). Fig. 16 presents a comparison of the predictions from (14) with the experimental measurement of the oscillator phase at $N f_{i n}$. Note that there is an excellent agreement, since the two curves are nearly superimposed. At the lower offset frequencies, the phase noise is $20 \log (30)$ higher than the one at the lower harmonic frequency of the input source, included in the figure. The phase-noise spectrum in free-running conditions is also shown for comparison. The spectrum of the injection-locked oscillator follows the input-noise source up to its corner frequency $f_{3 \mathrm{~dB}} \cong 1.5 \mathrm{MHz}$. Up to this frequency there is a negligible effect of the circuit own noise sources. At low frequency offset there is an improvement of more than 30 dB. To obtain a lower spectral density at higher offset frequencies an input source with lower phase noise should be used.

\section{B) Case $\boldsymbol{P} \leq \boldsymbol{N}$}

For $P<N$ the quantities $A_{p} / V_{s}^{2}$ and $\Omega_{3 \mathrm{~dB}}$, will be identified from envelope-domain simulations through a numerical procedure. To obtain the frequency response of the oscillator phase with 
respect to the equivalent noise source $\left|I_{N}\right|^{2}$, this noise source will be replaced with a deterministic tone at the frequency $\Omega$. Then, a two-tone envelope-domain analysis will be carried out, using the frequency basis $\left(\omega_{1}=\omega_{o} / P, \omega_{2}=\Omega\right)$. Then, for each $\Omega$, the phase shift the phase shift will be calculated from the voltage spectrum at the observation node [21], doing:

$$
\Delta \phi\left(N \omega_{i n}+\Omega\right)=\frac{V^{*}\left(N \omega_{i n}-\Omega\right) e^{j \phi_{s}}-V\left(N \omega_{i n}+\Omega\right) e^{-j \phi_{s}}}{2 V\left(N \omega_{i n}\right)}
$$

To obtain the voltage components in the above expression, the time-varying harmonic components resulting from the envelope-domain analysis can be processed to provide:

$$
V\left(N \omega_{i n}+\Omega\right)=\left\langle X_{P, 1}(t)\right\rangle, \quad V\left(N \omega_{i n}-\Omega\right)=\left\langle X_{P,-1}(t)\right\rangle, \quad V\left(N \omega_{i n}\right)=\left\langle X_{P, 0}(t)\right\rangle
$$

where the operator \langle\rangle applies an averaging in the period $T_{i n}=1 / f_{i n}$ and $X_{k, l}(t)$ is the harmonic component corresponding to the frequency $k \omega_{1}+l \omega_{2}$. Note that the time-varying nature of the harmonics $X_{k, l}(t)$ is due to the presence of frequency components at multiples of the injection frequency $f_{\text {in }}$, which are removed by the operator \langle\rangle . Sweeping $\Omega$ and tracing $\left|\Delta \phi\left(N \omega_{i n}+\Omega\right)\right|^{2}$ versus $\Omega$ one obtains a frequency response from which one can identify the two quantities $A_{p} / V_{s}^{2}$ and $\Omega_{3 \mathrm{~dB}}$. Once these quantities are available, one can obtain the phase-noise spectrum through (17).

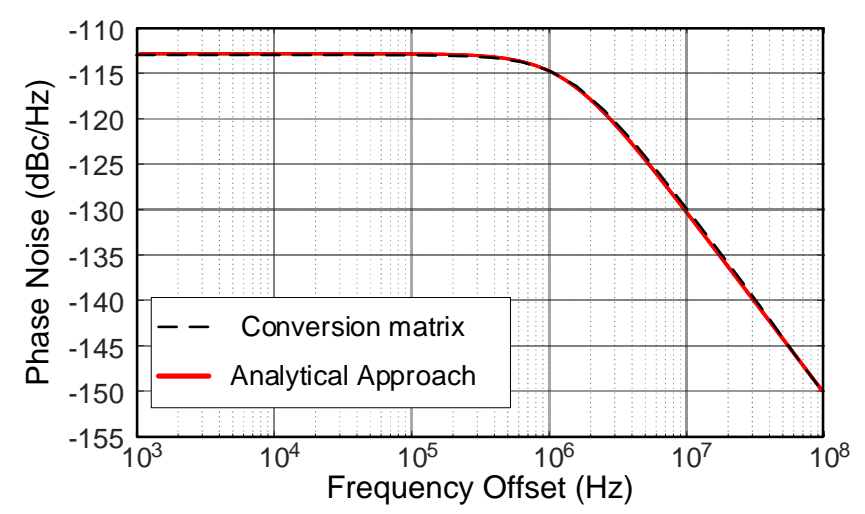

Fig. 15. Comparison of the results of the semi-analytical expression (14) and the conversionmatrix approach when only white-noise sources are considered. Note that the spectral density of the white-noise source $\left|I_{N}\right|^{2}$ was fitted in the standalone free-running oscillation. 


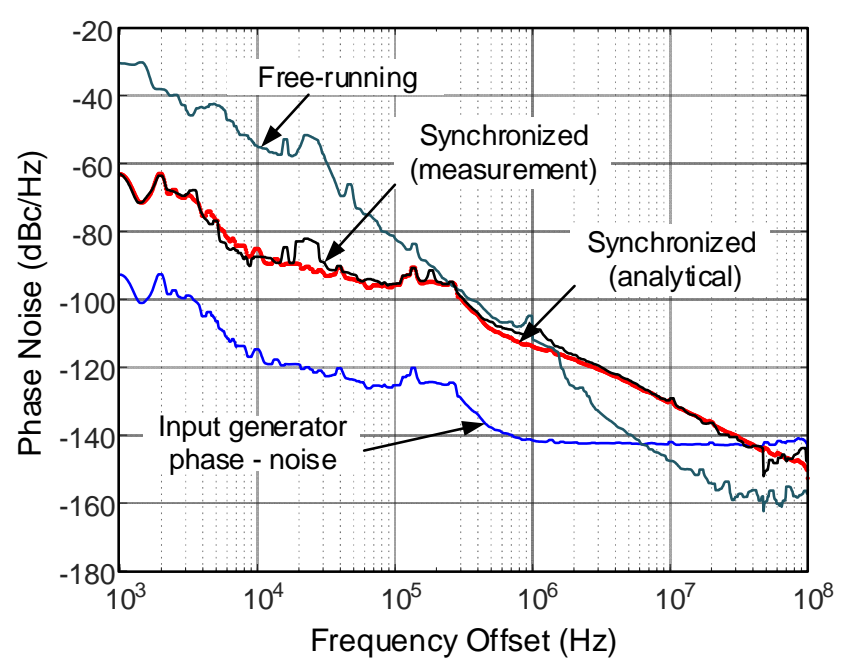

Fig. 16. Comparison of the predictions from (14) with the experimental measurement of the oscillator phase at $N f_{\text {in. }}$. At the lower offset frequencies, the phase noise is $20 \log (30)$ higher than the one at the lower harmonic frequency of the input source, included in the figure. The phasenoise spectrum in free-running conditions is also shown for comparison.

\section{CONCLUSION}

A methodology for the analysis of subharmonically injection-locked oscillators under a high order $N$ has been presented. This analysis is involved since harmonic-balance will not be applicable in most cases due to the need to fulfil the injection-locked oscillation condition under a high number of harmonic terms. Instead, an envelope-domain analysis has been proposed here. The procedure has been initially derived and tested in the case of an oscillator injected by a number of closely spaced input tones. In these conditions, the oscillator is described with the aid of a semi-analytical formulation, based on the modelling of this oscillator with an outer-tier admittance function extracted from harmonic-balance simulations. The locking bands about each of the input tones, obtained when sweeping a tuning voltage, can be efficiently predicted by averaging the phase of the integrated solution. This procedure has been extended to the more demanding case of a subharmonic injection-locked oscillator, simulated through a circuit-level envelope transient. The phase noise of the subharmonic injection-locked oscillator has been analysed with a semi-analytical formulation, based on an admittance model of the oscillator circuit in injection-locked conditions. The effect of the phase noise of an arbitrary periodic input waveform has been derived from its associated timing jitter. The analytical expression for the phase-noise spectral density shows that the injection-locked oscillator exhibits a low-pass response with respect to the noise sources. It initially follows $N$ times the phase noise of the fundamental frequency of the input-source and then follows its own input noise sources. The procedure has been illustrated with a practical FET-based subharmonic-injection locked oscillator at the order $N=30$. 


\section{ACKNOWLEDGEMENT}

This work was supported by the Spanish Ministry of Science, Innovation and Universities and the European Regional Development Fund (ERDF/FEDER) under the research project TEC2017-88242-C3-1-R.

\section{REFERENCES}

[1] C. Jany, A. Siligaris, P. Ferrari and P. Vincent, "A novel programmable harmonic selection technique based on the pseudo-locking of an oscillator by periodically repeated oscillations train," 44 ${ }^{\text {th }}$ European Microwave Conference (EuMC), Rome, Italy, 2014, pp. 1020-1023.

[2] C. Jany, A. Siligaris, J. L. Gonzalez-Jimenez, P. Vincent and P. Ferrari, "A Programmable Frequency Multiplier-by-29 Architecture for Millimeter Wave Applications," IEEE J. Solid-State Circuits, vol. 50, no. 7, pp. 1669-1679, Jul., 2015.

[3] C. Jany, A. Siligaris, P. Ferrari and P. Vincent, "A novel harmonic selection technique based on the injection of a periodically repeated oscillations train into an oscillator," IEEE MTT-S Int. Microw. Symp. (IMS2014), Tampa, FL, 2014, pp. 1-3.

[4] C. Jany, Conception et étude d'une synthèse de fréquence innovante en technologies CMOS avancées pour les applications en bande de fréquence millimétrique, Ph.D. Thesis, Grenoble University, September 2014.

[5] A. Boulmirat, C. Jany, A. Siligaris and J. L. G. Jimenez, "Low Power Locking Detector for Frequency Calibration of Multi-Frequency Injection Locked Oscillators," 2018 14th Conference on Ph.D. Research in Microelectronics and Electronics (PRIME), Prague, 2018, pp. 189-192.

[6] Z. Iskandar, A. Siligaris, J. Lugo-Alvarez, E. Pistono and P. Ferrari, "A 270-to-300 GHz LO-Path Phase Shifting Architecture for Sub-mm-wave Phased Arrays," 2018 48th European Microwave Conference (EuMC), Madrid, 2018, pp. 804-806.

[7] A. Siligaris, Z. Iskandar and J. M. Guerra, "Above 100-GHz low phase noise frequency generation in silicon integrated technologies," 2018 European Frequency and Time Forum (EFTF), Turin, 2018, pp. 32-36.

[8] K. S. Kundert, "Introduction to RF simulation and its application," IEEE J. Solid-State Circuits, vol. 34, no. 9, pp. 1298-1319, Sep 1999.

[9] K. Kundert, "Introduction to RF simulation and its application," Proc. IEEE Bipolar/BiCMOS Circuits Technol. Meeting, 1998, pp. 67-78.

[10] A. Suárez, Analysis and Design of Autonomous Microwave Circuits. Hoboken, NJ: Wiley IEEE Pres, 2009.

[11] A. Suárez, R. Quéré, Stability Analysis of Nonlinear Microwave Circuits. Boston, MA: Artech House, 2003.

[12] R. Quere, E. Ngoya, M. Camiade, A. Suarez, M. Hessane and J. Obregon, "Large signal design of broadband monolithic microwave frequency dividers and phase-locked oscillators," IEEE Transactions on Microwave Theory and Techniques, vol. 41, no. 11, pp. 1928-1938, Nov. 1993. 
[13] F. Ramirez, M. E. de Cos and A. Suarez, "Nonlinear analysis tools for the optimized design of harmonic-injection dividers," IEEE Trans. Microw. Theory Techn., vol. 51, no. 6, pp. 1752-1762, June 2003.

[14] S. Hernández, M. Pontón, A. Suárez, "Simulation Method for Complex Multivalued Curves in Injection-Locked Oscillators," IEEE Trans. Microw. Theory Techn., vol. 65, no. 11, pp. 4046-4062, Nov., 2017.

[15] S. Hernández, M. Pontón, S. Sancho and A. Suárez, "Analysis of high-order subharmonically injection-locked oscillators," 2019 49th European Microwave Conference (EuMC), Paris, France, 2019, pp. 244-247.

[16] E. Ngoya and R. Larcheveque, "Envelop transient analysis: a new method for the transient and steady state analysis of microwave communication circuits and systems," IEEE MTTS Int. Microwave Symp. Dig., San Francisco, CA, USA, 1996, vol.3, pp. 1365-1368.

[17] E. Ngoya, J. Rousset, and D. Argollo, "Rigorous RF and microwave oscillator phase noise calculation by the envelope transient technique," IEEE MTT-S Int. Microwave Symp. Dig., 2000, pp. 90-94.

[18] J. C. Pedro and N. B. Carvalho, "Simulation of RF circuits driven by modulated signals without bandwidth constraints," IEEE MTT-S Int. Microwave Symp. Dig., Seattle, WA, USA, 2002, pp. 2173-2176 vol.3.

[19] N. B. Carvalho, J. C. Pedro, W. Jang and M. B. Steer, "Nonlinear RF circuits and systems simulation when driven by several modulated signals," IEEE Trans. Microw. Theory Techn, vol. 54, no. 2, pp. 572-579, Feb. 2006.

[20] E. Cos, A. Suarez and S. Sancho, "Envelope transient analysis of self-oscillating mixers," IEEE Trans. Microw. Theory Tec, vol. 52, no. 4, pp. 1090-1100, April 2004.

[21] F. Ramirez, M. Ponton, S. Sancho and A. Suarez, "Phase-Noise Analysis of InjectionLocked Oscillators and Analog Frequency Dividers," IEEE Trans. Microw. Theory Techn, vol. 56, no. 2, pp. 393-407, Feb. 2008.

[22] A. Suarez, F. Ramirez and S. Sancho, "Stability and Noise Analysis of Coupled-Oscillator Systems," IEEE Trans. Microw. Theory Techn., vol. 59, no. 4, pp. 1032-1046, Apr. 2011.

[23] S. Sancho, M. Ponton and A. Suarez, "Effects of Noisy and Modulated Interferers on the Free-Running Oscillator Spectrum," IEEE Trans. Microw. Theory Techn., vol. 66, no. 4, pp. 1831-1842, April 2018.

[24] F. Ramírez, S. Sancho, M. Pontón and A. Suárez, "Analysis of Chirped Oscillators under Injection Signals," IEEE MTT-S Int. Microw. Symp - IMS, Philadelphia, PA, 2018, pp. 172-175.

[25] F. Ramírez, S. Sancho, M. Pontón, A. Suárez, "Two-Scale Envelope-Domain Analysis of Injected Chirped Oscillators," IEEE Trans. Microw. Theory Techn., vol. 66, no. 12, pp. 5449-5461, Dec., 2018.

[26] E. Fernández, F. Ramírez, A. Suárez, S. Sancho, "Stability and phase-noise analysis of pulsed injection-locked oscillators," IEEE Trans. Microw. Theory Techn., vol. 61, no. 1, pp. 482-491, Jan., 2013.

[27] J. Guckenheimer and P. Holmes, Nonlinear Oscillations, Dynamical Systems and Bifurcations of Vector Fields. New York, NY, USA: Springer-Verlag, 1990.

[28] J. M. T. Thompson and H. B. Stewart, Nonlinear Dynamics and Chaos, 2nd ed. New York: Wiley 
[29] A. Suarez, E. Fernandez, F. Ramirez and S. Sancho, "Stability and Bifurcation Analysis of Self-Oscillating Quasi-Periodic Regimes," IEEE Trans. Microw. Theory Techn, vol. 60, no. 3, pp. 528-541, March 2012.

[30] J. M. Paillot, J. C. Nallatamby, M. Hessane, R. Quere, M. Prigent, and J. Rousset, "General program for steady state, stability, and FM noise analysis of microwave oscillators," IEEE MTT-S Int. Microw. Symp., 1990, vol. 3, pp. 1287-1290.

[31] V. Rizzoli, F. Mastri, and D. Masotti, "General noise analysis of nonlinear microwave circuits by the piecewise harmonic-balance technique," IEEE Trans. Microw. Theory Tech., vol. 42, no. 5, pp. 807-819, May 1994.

[32] J. C. Nallatamby, M. Prigent, J. C. Sarkissian, R. Quéré, and J. Obregón, “A new approach to nonlinear analysis of noise behavior of synchronized oscillators and analog-frequency dividers," IEEE Trans. Microw. Theory Tech., vol. 46, no. 8, pp. 1168-1171, Aug. 1998.

[33] F. X. Kaertner, "Analysis of white and $\mathrm{f}^{-\alpha}$ noise in oscillators", Int. J. Circuit Theory Appl., vol. 18, pp. 485-519, 1990.

[34] M.Takahashi, K. Ogawa, K.S. Kundert, "VCO Jitter Simulation and Its Comparison with Measurement", Design Automation Conf., 1999. Proc. of the ASP-DAC '99. Asia and South Pacific, vol. 1, pp.85-88, Jan. 1999.

\section{Bibliographies}

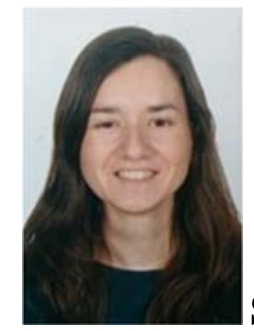

Silvia Hernández was born in the Canary Islands, Spain. She received her M.S. degree in Telecommunication Engineering from the University of Las Palmas de Gran Canaria, Canary Islands, Spain, in 2015. The same year, she entered the Institute for the Technological Development and Innovation in Communications, University of Las Palmas de Gran Canaria, as a research assistant. In 2016, she joined the Communications Engineering Department, University of Cantabria, where she is currently working towards her Ph.D. degree.

Her research interests include stability analysis and the study of new methods for the analysis and design of nonlinear microwave circuits.

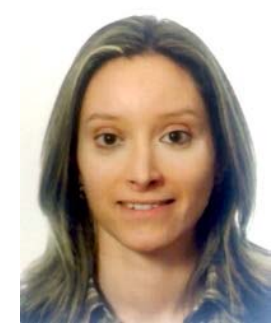

Mabel Pontón was born in Santander, Spain. She received the bachelor's degree in telecommunication engineering, master's degree in information technologies and wireless communications systems, and $\mathrm{Ph} . \mathrm{D}$. degree from the University of Cantabria, Santander, in 2004, 2008, and 2010, respectively. 
In 2006, she joined the Communications Engineering Department, University of Cantabria. From 2011 to 2013, she was with the Group of Electronic Design and Applications, Georgia Institute of Technology, Atlanta, GA, USA, as a Post-Doctoral Research Fellow.

Her current research interests include the nonlinear analysis and simulation of radiofrequency and microwave circuits, with an emphasis on phase-noise, stability, and bifurcation analysis of complex oscillator topologies.

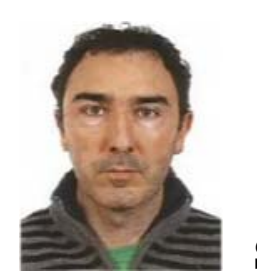

Sergio Sancho received the degree in Physics from Basque Country University in 1997. In 1998 he joined the Communications Engineering Department of the University of Cantabria, Spain, where he received the Ph.D. degree in Electronic Engineering in February 2002. At present, he works at the University of Cantabria, as an Associate Professor of its Communications Engineering Department. His research interests include the nonlinear analysis of microwave autonomous circuits and frequency synthesizers, including stochastic and phase-noise analysis.

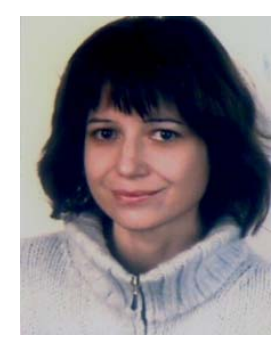

Almudena Suárez was born in Santander, Spain. She received the degree in electronic physics and the Ph.D. degree from the University of Cantabria, Santander, Spain, in 1987 and 1992, respectively, and the Ph.D. degree in electronics from the University of Limoges, France, in 1993. At present, she is a Full Professor at the University of Cantabria, and a member of its Communications Engineering Department. She has authored the book Analysis and design of autonomous microwave circuits for the publisher IEEE-Wiley and co-authored the book Stability analysis of microwave circuits for the publisher Artech-House. She belongs to the technical committees of the IEEE International Microwave Symposium and European Microwave Conference. She was an IEEE Distinguished Microwave Lecturer for the period 2006-2008. She is an associate editor of the IEEE Microwave Magazine. She is a member of the Board of Directors of EuMA and the Editor in Chief of International Journal of Microwave and Wireless Technologies from Cambridge University Press.

\section{List of figures and tables}

Fig. 1. Calculation of the derivatives of the oscillator nonlinear-admittance function through finite differences in harmonic balance, using an auxiliary generator. 
Fig. 2. FET-based (ATF-34143) oscillator at $f_{o}=2.7 \mathrm{GHz}$. The frequency is tuned with the varactor diode SMV1235. (a) Schematic. (b) Photograph.

Fig. 3. Spectrum obtained through (3) and with envelope transient for the spectral-line power $P_{i n}=-33 \mathrm{dBm}$ and frequency spacing $\Delta f=16 \mathrm{MHz}$.

Fig. 4. Behavior under six input lines, between $2.7 \mathrm{GHz}$ and $2.78 \mathrm{GHz}$, with $\Delta f=16 \mathrm{MHz}$. (a) Time variation of the phase shift for three $V_{D}$ values, within the first synchronization band $\left(f_{1}=2.7 \mathrm{GHz}\right)$. (b) Average value of $-\phi(t)$ versus $V_{D}$.

Fig. 5. Behavior under six input lines, between $2.7 \mathrm{GHz}$ and $2.78 \mathrm{GHz}$, with $\Delta f=16 \mathrm{MHz}$. Average value of $\Delta V(t)$ versus $V_{D}$. Three synchronization bands can be distinguished with an ellipsoidal form.

Fig. 6. Behavior under six input lines, between $2.7 \mathrm{GHz}$ and $2.78 \mathrm{GHz}$, with $\Delta f=16 \mathrm{MHz}$. Detection of synchronization bands from the analysis of the solution spectrum. (a) Frequencies of the spectral line with larger output power and its neighboring spectral lines, traced versus $V_{D}$. (b) Output power at the frequencies of the input tones.

Fig. 7. Measurement results. (a) Input tones. (b) Unlocked spectrum for $V_{D}=0.28 \mathrm{~V}$. (c) Synchronization to $f_{2}$ for $V_{D}=1.69 \mathrm{~V}$. (d) $f_{4}$ for $V_{D}=2.85 \mathrm{~V}$.

Fig. 8. Envelope-domain analysis of the high-order subharmonic injection-locked oscillator. Different choices of the fundamental frequency $f_{o} / P$ in the Fourier-series representation of the circuit variables.

Fig. 9. Injection locking by a pulsed signal at $90 \mathrm{MHz}$. Output spectrum at $f_{\text {out }}=30 \times 90 \mathrm{MHz}$ when considering $P=1,4,6$. HB results are superimposed.

Fig. 10.HB analysis of the circuit in Fig. 2 under a rectangular input signal of $V_{\text {peak }}=1 \mathrm{~V}$, frequency $90 \mathrm{MHz}$ and duty cycle $25 \%$. (a) Without AG. The oscillation is not excited. (b) With an $\mathrm{AG}$, after fulfilment of the optimization condition $Y(V, \phi)=0$. The injection-locked oscillation is clearly visible.

Fig. 11. Locking band at $N=30$. (a) Synchronization curve, versus $V_{D}$, obtained with HB. Experimental points are superimposed. (b) Result of the phase averaging $\left\langle\phi_{P}(t)\right\rangle=\phi_{o, P}$. (c) Result of averaging the amplitude of $P$-th harmonic component of the output voltage, denoted as $\left\langle V_{P}(t)\right\rangle=V_{o, P}$.

Fig. 12. Oscillator locking to different spectral lines of the input source by tuning $V_{D}$. (a) Locking to $f_{1}=2.7 \mathrm{GHz}(N=30)$. (b) Locking to $f_{2}=2.79 \mathrm{GHz}(N+1)$. (c) Locking to $f_{3}=2.88$ $\mathrm{GHz}(N+2)$.

Fig. 13. Increase of the frequency selectivity through the use of a second oscillator. (a) Simulations. (b) Measurements.

Fig. 14. Fitting the spectral density of the original free-running oscillator with a single equivalent current source of spectral density $\left|I_{N}(\Omega)\right|^{2}$.

Fig. 15. Comparison of the results of the semi-analytical expression (14) and the conversionmatrix approach when only white-noise sources are considered. Note that the spectral density of the white-noise source $\left|I_{N}\right|^{2}$ was fitted in the standalone free-running oscillator.

Fig. 16. Comparison of the predictions from (14) with the experimental measurement of the oscillator phase at $N f_{\text {in }}$. 